\title{
Ubiquity and large intersections properties under digit frequencies constraints
}

\author{
BY JULIEN BARRAL \\ Projet SISYPHE - INRIA Rocquencourt, B.P. 105 \\ 78153 Le Chesnay Cedex, FRANCE \\ AND STÉPHANE SEURET \\ LAMA - Université Paris 12 - UFR Sciences et Technologie \\ 61, avenue du Général de Gaulle, 94010 Créteil Cedex, FRANCE \\ (Received )
}

\begin{abstract}
We are interested in two properties of real numbers: the first one is the property of being well-approximated by some dense family of real numbers $\left\{x_{n}\right\}_{n \geq 1}$, such as rational numbers and more generally algebraic numbers, and the second one is the property of having given digit frequencies in some $b$-adic expansion.

We combine these two ways of classifying the real numbers, in order to provide a finer classification. We exhibit sets $S$ of points $x$ which are approximated at a given rate by some of the $\left\{x_{n}\right\}_{n}$, those $x_{n}$ being selected according to their digit frequencies. We compute the Hausdorff dimension of any countable intersection of such sets $S$, and prove that these sets enjoy the so-called large intersection property.
\end{abstract}

\section{Introduction}

Ubiquity theory starts with the following question. Consider a $\sigma$-compact metric space $(X, \rho)$, a dense sequence $\left(x_{n}\right)_{n \geq 1}$ in $X$ and a positive sequence $\left(l_{n}\right)_{n \geq 1}$ such that for each compact subset $K$ of $X, \lim _{n \rightarrow \infty, x_{n} \in K} l_{n}=0$. What is for $\delta>0$ the Hausdorff dimension of the limsup set $S_{\delta}=\lim \sup _{n \rightarrow \infty} B\left(x_{n}, l_{n}^{\delta}\right)$ ? $(B(x, r)$ stands for the open ball of radius $r$ centered at $x$ ).

The first answer to a ubiquity question has been given in the context of Diophantine approximation. The dense sequence $\left(x_{n}\right)_{n \geq 1}$ is constituted by the rational numbers asso-

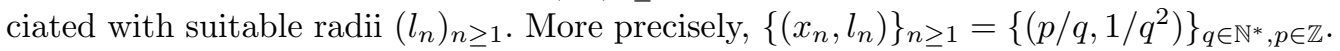
Jarnick proved in $[\mathbf{2 4}]$ that the Hausdorff dimension of the set $S_{\delta}$ defined by

$$
S_{\delta}=\left\{x \in \mathbb{R}:\left\{\begin{array}{l}
\exists\left(p_{n}, q_{n}\right)_{n} \in\left(\mathbb{Z} \times \mathbb{N}^{*}\right)^{\mathbb{N}} \text { such that } q_{n} \rightarrow+\infty \\
p_{n} / q_{n} \text { irreducible and }\left|x-p_{n} / q_{n}\right|<\left(1 / q_{n}^{2}\right)^{\delta}
\end{array}\right\}\right.
$$

is equal to $1 / \delta$ when $\delta>1$ (Dirichlet proved before that $S_{1}=\mathbb{R}$ ). Similar results were obtained in $\mathbb{R}^{d}$ for various families $\left\{\left(x_{n}, l_{n}\right)\right\}_{n \geq 1}[\mathbf{8}, \mathbf{9}, \mathbf{1}, \mathbf{2 3}]$ or in metric spaces enjoying enough self-similar properties to support a monofractal measure $[\mathbf{1 3}, \mathbf{1 4}, \mathbf{4}]$, like the middle third Cantor set $[\mathbf{2 6}]$. 
One motivation for looking at the sets $S_{\delta}$ is that they provide a classification of the elements of $X$ according to their approximation degree by the family $\left\{\left(x_{n}, l_{n}\right)\right\}_{n \geq 1}$, where the approximation degree $\delta_{x}$ of $x$ is defined by

$$
\delta_{x}=\sup \left\{\delta>0: x \in S_{\delta}\right\}
$$

(with the convention sup $\emptyset=0$ ).

From now on we work with $X=\mathbb{R}$. We shall use repetitively the following notion of approximation family.

Definition 1.1. Let $\left(x_{n}\right)_{n \geq 1}$ be a sequence of real numbers dense in $\mathbb{R}$, and $\left(l_{n}\right)_{n \geq 1}$ a sequence of positive real numbers.

When $\lim _{n \rightarrow \infty, x_{n} \in K} l_{n}=0$ for each compact subinterval $K$ of $\mathbb{R}$, the family of pairs $\widetilde{X}=\left\{\left(x_{n}, l_{n}\right)\right\}_{n \geq 1}$ is called an approximation family in $\mathbb{R}$.

In $\mathbb{R}$, an alternative to the classification by the approximation degree associated with some approximation family $\widetilde{X}=\left\{\left(x_{n}, l_{n}\right)\right\}_{n \geq 1}$ is, given an integer basis $b \geq 2$, to consider the asymptotic behavior of digit frequencies in the $b$-adic expansions of real numbers $[\mathbf{6}, \mathbf{1 5}]$. Let us recall the definition of digit frequencies.

Given an integer $b \geq 2$, for any $x \in \mathbb{R}$, consider the $b$-adic expansion of the fractional part of $x$, denoted $\{x\}:\{x\}=\sum_{m=1}^{\infty} x_{m} b^{-m}$, where $\forall m, x_{m} \in\{0,1, \ldots, b-1\}$. Consider then the mappings $\phi_{i, n}(x)$ (for every $i \in\{0,1, \ldots, b-1\}$ and $n \geq 1$ )

$$
x \mapsto \phi_{i, n}(x)=\frac{\#\left\{m \leq n: x_{m}=i\right\}}{n} .
$$

It is well-known that for Lebesgue-almost every real number $x \in \mathbb{R}$, for all $i \in\{0, . ., b-1\}$, the limit frequency of the digit $i$ exists and $\lim _{n \rightarrow+\infty} \phi_{i, n}(x)=1 / b$. In addition, given a probability vector $\tilde{\pi}=\left(\pi_{0}, \pi_{1}, \ldots, \pi_{b-1}\right) \in[0,1]^{b}$ (i.e. $\sum_{i=0}^{b-1} \pi_{i}=1$ ), Besicovitch and later Eggleston $[\mathbf{6}, \mathbf{1 5}]$ investigated the set $A(\tilde{\pi})$ of points $x$ such that the limit frequency of the digit $i$ in the $b$-adic expansion of $x$ is equal to $\pi_{i}$, for all $i \in\{0,1, \ldots, b-1\}$ :

$$
A(\widetilde{\pi})=\left\{x \in \mathbb{R}: \forall i \in\{0,1, \ldots, b-1\}, \lim _{n \rightarrow+\infty} \phi_{i, n}(x)=\pi_{i}\right\} .
$$

They proved that (dim stands for the Hausdorff dimension)

$$
\operatorname{dim} A(\tilde{\pi})=\alpha(\widetilde{\pi}) \quad \text { with } \quad \alpha(\widetilde{\pi})=\sum_{i=0}^{b-1}-\pi_{i} \log _{b} \pi_{i} .
$$

In this paper, we combine these two viewpoints in order to provide a finer classification inside the sets $S_{\delta}$, by using digit frequencies in the $b$-adic expansion of the terms of the dense sequence of real numbers $\left(x_{n}\right)_{n \geq 1}$. Subsequently, we highlight the relationship between the digit frequencies in the $b$-adic expansion of a real number $x$ and the approximation degree of $x$ by a family $\left\{\left(x_{n}, l_{n}\right)\right\}_{n \geq 1}$.

Our approach is based on the fact that the set of points having given asymptotic digit frequencies carries a self-similar measure. To see this, consider the so-called multinomial measure $\mu_{\tilde{\pi}}$ associated with $\tilde{\pi}$, i.e. the unique self-similar probability measure such that $\mu_{\widetilde{\pi}}(\cdot)=\sum_{k=0}^{b-1} \pi_{k} \mu_{\widetilde{\pi}}(b \cdot-k)$. We have $\mu_{\widetilde{\pi}}(A(\widetilde{\pi}))=1$ and

$$
\lim _{r \rightarrow 0^{+}} \frac{\log \mu_{\tilde{\pi}}(B(x, r))}{\log (r)}=\alpha(\widetilde{\pi}) \text { for } \mu_{\tilde{\pi}} \text {-a.e. } x .
$$

Then, classical arguments imply (1.4) (see Lemma $2 \cdot 1$ in Section $2 \cdot 2$ ). 
The previous discussion leads us to consider the following limsup sets.

Definition $1 \cdot 2$. Let $\left.\tilde{X}=\left\{\left(x_{n}, l_{n}\right)\right\}\right)_{n \geq 1}$ be an approximation family in $\mathbb{R}, b \geq 2$ an integer, $\widetilde{\pi}$ a probability vector in $(0,1)^{b}$ and $\mu_{\tilde{\pi}}$ the associated multinomial measure. Let $\widetilde{\varepsilon}=\left(\varepsilon_{n}\right)_{n \geq 1}$ be a positive non-increasing sequence converging to zero, and $\delta \geq 1$.

Consider the property

$$
\mathcal{C}\left(\tilde{\pi}, x_{n}, l_{n}, \varepsilon_{n}\right)=\left\{\begin{array}{l}
\left(l_{n}\right)^{\alpha(\widetilde{\pi})+\varepsilon_{n}} \leq \mu_{\widetilde{\pi}}\left(B\left(\left\{x_{n}\right\}, l_{n}\right)\right) \leq\left(l_{n}\right)^{\alpha(\widetilde{\pi})-\varepsilon_{n}} \text { and } \\
\max _{0 \leq i \leq b-1}\left(\sup _{x \in B\left(x_{n}, l_{n}\right)}\left|\phi_{i,\left[-\log _{b} l_{n}\right]}(\{x\})-\pi_{i}\right|\right) \leq \varepsilon_{n}
\end{array}\right.
$$

where $[y]$ stands for the integer part of $y \in \mathbb{R}$. Finally set

$$
\mathcal{S}(\tilde{X}, \tilde{\pi}, \delta, \widetilde{\varepsilon})=\bigcap_{N \geq 1} \bigcup_{n \geq N: \mathcal{C}\left(\tilde{\pi}, x_{n}, l_{n}, \varepsilon_{n}\right)} \operatorname{\bigcup holds}_{n}\left(x_{n}-l_{n}^{\delta}, x_{n}+l_{n}^{\delta}\right),
$$

This set contains the real numbers $x$ which are infinitely often approximated at rate $\delta$ by some of the $x_{n}$ relatively to the radii $l_{n}$, those $x_{n}$ being selected according to the fact that $\frac{\log \mu_{\widetilde{\pi}}\left(B\left(\left\{x_{n}\right\}, l_{n}\right)\right)}{\log \left(l_{n}\right)}$ tends to $\alpha(\widetilde{\pi})$ and $\sup _{x \in B\left(x_{n}, l_{n}\right)} \phi_{i,\left[-\log _{b} l_{n}\right]}(\{x\})$ tends to $\pi_{i}$ for every $i \in\{0, \ldots, b-1\}$ as $n \rightarrow+\infty$. The dependence of $\mathcal{S}(\widetilde{X}, \widetilde{\pi}, \delta, \widetilde{\varepsilon})$ on $b$ is hidden in the condition $\mathcal{C}\left(\tilde{\pi}, x_{n}, l_{n}, \varepsilon_{n}\right)$.

REMARK 1·3. (1) It is tempting to consider the smaller sets of the form $S_{\delta} \cap A(\widetilde{\pi})$. Nevertheless, these sets cannot be expressed as limsup sets, so they are not associated with "ubiquitous systems" anymore. Moreover, when $\pi$ has positive entries and $\left\{\left(x_{n}, l_{n}\right)\right\}_{n \geq 1}$ is taken equal to $\left\{\left(k b^{-j}, b^{-j}\right)\right\}_{k \in \mathbb{Z}, j \geq 0}$, it can be shown that $S_{\delta} \cap A(\tilde{\pi})$ is empty when $\delta$ is large enough. Thus, although the sets $S_{\delta} \cap A(\widetilde{\pi})$ deserve to be considered, their Hausdorff dimension seems difficult to reach in full generality.

(2) A simple way to construct approximation families consists in taking a dense sequence $\left(y_{p}\right)_{p \geq 1}$ in $[0,1]$ as well as a non-decreasing sequence $\left(\lambda_{p}\right)_{p \geq 1}$, and then to take

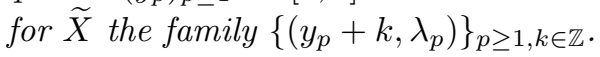

Our goal is to compute the Hausdorff dimension of any countable intersection of such sets $\mathcal{S}(\widetilde{X}, \widetilde{\pi}, \delta, \widetilde{\varepsilon})$. We also show that, under suitable assumptions, such an intersection provides a new type of limsup set enjoying the remarkable property introduced by Falconer in $[\mathbf{1 6}, \mathbf{1 7}]$, called "large intersection property", and defined as follows.

Definition 1.4. [17] For $0<\alpha \leq 1$, the class $\mathcal{G}^{\alpha}$ of sets with large intersection consists in the $G_{\delta}$-subsets $\Omega \subset \mathbb{R}$ such that for all sequences of similarity transformations of $\mathbb{R}\left(f_{k}\right)_{k \in \mathbb{N}}, \operatorname{dim}\left(\bigcap_{k \in \mathbb{N}} f_{k}(\Omega)\right) \geq \alpha$.

In order to get accurate bound estimations for the Hausdorff dimension of subsets of $\mathbb{R}$, two assumptions are needed on the covering of $\mathbb{R}$ by the intervals $B\left(x_{n}, l_{n}\right)$.

The first assumption is sufficient to obtain a lower bound for $\operatorname{dim} \mathcal{S}(\widetilde{X}, \widetilde{\pi}, \delta, \widetilde{\varepsilon})$. It requires that

$$
\mathbb{R}=\limsup _{n \in \mathbb{N}} B\left(x_{n}, l_{n} / 2\right) .
$$

The second assumption, almost as simple as (1.8), concerns the covering's optimality 
and is useful when looking for sharp upper bounds for $\operatorname{dim} \mathcal{S}(\widetilde{X}, \widetilde{\pi}, \delta, \widetilde{\varepsilon})$. For every $j \geq 0$, let

$$
T_{j}=\left\{n: 2^{-(j+1)}<l_{n} \leq 2^{-j}\right\} .
$$

DEFINITION 1.5. The family $\left\{\left(x_{n}, l_{n}\right)\right\}_{n \geq 1}$ is said to form a weakly redundant approximation family (WRA family) if there is a sequence of integers $\left(N_{j}\right)_{j \geq 0}$ such that

(i) $\lim _{j \rightarrow \infty} \log _{2} N_{j} / j=0$.

(ii) for every $j \geq 1, T_{j}$ can be decomposed into $N_{j}$ pairwise disjoint subsets of integers denoted $T_{j, 1}, \ldots, T_{j, N_{j}}$ such that for each $1 \leq i \leq N_{j}$, the family $\left\{B\left(x_{n}, l_{n}\right): n \in\right.$ $\left.T_{j, i}\right\}$ is composed of disjoint balls.

Heuristically, a WRA family satisfies that asymptotically a point $x \in \mathbb{R}$ cannot belong to more than $2^{o(j)}$ balls $B\left(x_{n}, l_{n}\right)$ such that $l_{n} \approx 2^{j}$.

The covering property (1.8) and weak redundancy together ensure that the family $\widetilde{X}=$ $\left\{\left(x_{n}, l_{n}\right)\right\}_{n \geq 1}$ is quite homogeneously distributed in $\mathbb{R}$. Examples of remarkable families $\widetilde{X}$ are discussed in Section 6. We remark that the family $\left\{\left(p / q, 1 / q^{2}\right)\right\}_{p \in \mathbb{Z}, q \in \mathbb{N}^{*}, p \wedge q=1}$ is WRA, while the whole family $\left\{\left(p / q, 1 / q^{2}\right)\right\}_{p \in \mathbb{Z}, q \in \mathbb{N}^{*}}$ is not.

We now state the main result of this paper.

TheOREM 1.6. Let $\widetilde{X}=\left\{\left(x_{n}, l_{n}\right)\right\}_{n \geq 1}$ be an approximation family in $\mathbb{R}$. We consider:

- $\widetilde{B}=\left(b_{p}\right)_{p \geq 1}$ a sequence of integers $\geq 2$,

- $\widetilde{\Delta}=\left(\delta_{p}\right)_{p \geq 1}$ a sequence of real numbers $\geq 1$,

- $\widetilde{\Pi}=\left(\widetilde{\pi}^{p}\right)_{p \geq 1}$ a sequence of probability vectors $\widetilde{\pi}^{p}=\left(\pi_{0}^{p}, \pi_{1}^{p}, \ldots, \pi_{b_{p}-1}^{k}\right)$ in $(0,1)^{b_{p}}$,

- $\left(\mu_{\tilde{\pi}^{p}}\right)_{p \geq 1}$ the sequence of multinomial measures associated with $\left(\widetilde{\pi}^{p}\right)_{p \geq 1}$.

- $\widetilde{E}=\left(\widetilde{\varepsilon}^{p}\right)_{p \geq 1}$ a sequence of positive non-increasing sequences $\widetilde{\varepsilon}^{p}=\left(\varepsilon_{n}^{p}\right)_{n \geq 1}$ all converging to 0 when $n$ goes to infinity.

We define

$$
\mathcal{A}(\widetilde{X}, \widetilde{\Delta}, \widetilde{\Pi}, \widetilde{E})=\bigcap_{p \geq 1} \mathcal{S}\left(\widetilde{X}, \widetilde{\pi}^{p}, \delta_{p}, \widetilde{\varepsilon}^{p}\right) \quad \text { and } \quad \alpha(\widetilde{\Delta}, \widetilde{\Pi})=\inf _{p \geq 1} \frac{\alpha\left(\widetilde{\pi}^{p}\right)}{\delta_{p}} .
$$

(i) If $\underset{\widetilde{X}}{\widetilde{X}}$ is a WRA family, then $\operatorname{dim} \mathcal{A}(\widetilde{X}, \widetilde{\Delta}, \widetilde{\Pi}, \widetilde{E}) \leq \alpha(\widetilde{\Delta}, \widetilde{\Pi})$.

(ii) If $\widetilde{X}$ satisfies the covering property (1.8), the sequence $\widetilde{E}$ can be chosen so that $\mathcal{A}(\widetilde{X}, \widetilde{\Delta}, \widetilde{\Pi}, \widetilde{E}) \neq \emptyset$ and

$$
\operatorname{dim} \mathcal{A}(\widetilde{X}, \widetilde{\Delta}, \widetilde{\Pi}, \widetilde{E}) \geq \alpha(\widetilde{\Delta}, \widetilde{\Pi}) .
$$

(iii) Consequently, if $\widetilde{X}$ is a WRA family satisfying (1.8) and $\widetilde{E}$ is chosen as in part (ii), then (1.11) is an equality.

(iv) Let $\widetilde{E}$ be chosen as in part (ii). If $\alpha(\widetilde{\Delta}, \widetilde{\Pi})>0$, then $\mathcal{A}(\widetilde{X}, \widetilde{\Delta}, \widetilde{\Pi}, \widetilde{E})$ belongs to the class of sets with large intersection $\mathcal{G}^{\alpha(\widetilde{\Delta}, \widetilde{\Pi})}$.

REMARK 1.7. (1) The proof of Theorem 1.6 shows that condition (1.8) can be weakened by assuming that there exists an at most countable subset $D$ of $\mathbb{R}$ such that $\mathbb{R} \backslash D \subset$ $\limsup _{n \rightarrow \infty} B\left(x_{n}, l_{n} / 2\right)$.

(2) For $r>0$, let $\psi(r)=\sqrt{\frac{\log \log |\log (r)|}{|\log r|}}$. When $\widetilde{E}$ is finite, there exists $C>0$ such that a good choice for $\widetilde{E}$ in Theorem 1.6.(ii) is $\varepsilon_{n}^{p}=C \psi\left(l_{n}\right)$. When $\widetilde{E}$ is not finite, a convenient choice is $\varepsilon_{n}^{p}=\kappa\left(l_{n}\right) \psi\left(l_{n}\right)$, where $\kappa(r)$ verifies $\kappa(r)=o(\sqrt{\log \log |\log (r)|})$ 
and $\kappa(r)$ tends to $\infty$ as $r$ tends to 0 . For instance, $\kappa(r)=\log ^{\circ k}(|\log r|)$ with $k \geq 4$ is convenient ( $\mathrm{k}$ means $k$ th iteration).

In particular, in both cases, $\varepsilon_{n}^{p}$ does not depend on $p$.

In Theorem $1 \cdot 6$, only one family $\widetilde{X}$ is used. Since the the classes $\left\{\mathcal{G}^{s}\right\}_{s>0}$ are decreasing and each $\mathcal{G}^{s}$ is stable under countable intersection, without any effort we get:

Corollary 1.8. Consider a countable number of sets $\mathcal{A}_{i}=\mathcal{A}\left(\widetilde{X}_{i}, \widetilde{\Delta}_{i}, \widetilde{\Pi}_{i}, \widetilde{E}_{i}\right)(i \geq$ 1) associated with sets of parameters $\left(\widetilde{X}_{i}, \widetilde{\Delta}_{i}, \widetilde{\Pi}_{i}, \widetilde{E}_{i}\right)$ defined as in Theorem 1.6. If the families $\widetilde{X}_{i}$ are WRA and verify (1.8) and if the sequences $\widetilde{E}_{i}$ are chosen as in part (ii) of Theorem 1.6, then their intersection satisfies $\operatorname{dim} \bigcap_{i \geq 1} \mathcal{A}_{i}=\inf _{i \geq 1} \alpha\left(\widetilde{\Delta}_{i}, \widetilde{\Pi}_{i}\right)$.

We finally mention a possible extension of our results to limsup sets included in Cantor sets. It is natural to seek for a result similar to (1.11) after weakening the assumption that the coordinates of the vectors $\widetilde{\pi}^{p}$ are strictly positive. When all the measures $\mu_{\widetilde{\pi}^{p}}$ have the same support $S \subset \limsup _{n \in \mathbb{N}} B\left(x_{n}, l_{n} / 2\right)$, relation (1.11) still holds when some $\widetilde{\pi}^{i}$ equals 0 .

As a special case, we can take $S$ equal to the middle third Cantor set $\mathcal{C}_{1 / 3}$ and the family $\left\{\left(x_{n}, l_{n}\right)\right\}_{n \geq 1}$ equal to $\left\{\left(k 3^{-j}, 3^{-j}\right)\right\}_{j \geq 1, k \in E_{j}}$, where $E_{j}$ is the set of integers between 0 and $3^{j}$ such that $k 3^{-j} \in \mathcal{C}_{1 / 3}$. The measure $\mu_{\widetilde{\pi}^{p}}$ can be taken with support equal to $\mathcal{C}_{1 / 3}$. Then a straightforward extension of Theorem 1.6 yields a conjecture by Mahler, already proved in $[\mathbf{1 4}, \mathbf{2 6}]$. The problem was the existence and the size (in terms of Hausdorff dimension) of the irrational points of the Cantor set $\mathcal{C}_{1 / 3}$ whose approximation rate by the rational numbers $\delta_{x}$ is finite. In other words, does there exist irrational non-Liouville numbers in $\mathcal{C}_{1 / 3}$ ? Theorem $1 \cdot 6$, yields that some subsets of $\mathcal{C}_{1 / 3}$ constituted by real numbers approximated at given rates by the family $\left\{\left(k 3^{-j}, 3^{-j}\right)\right\}_{j \geq 1, k \in E_{j}}$ have positive Hausdorff dimensions. Consequently they contain non-Liouville irrational numbers. Our results go beyond this claim.

The theorem established in this paper are substantial improvements of the result announced in $[\mathbf{2}, \mathbf{3}]$.

\section{Tools}

\subsection{Recalls on generalized Hausdorff measures}

In the rest of the paper, the diameter of a subset $B$ of $\mathbb{R}$ is denoted $|B|$. Let $\xi$ be a gauge function, i.e. a non-negative non-decreasing function on $\mathbb{R}_{+}$such that $\lim _{x \rightarrow 0^{+}} \xi(x)=0$. Let $S$ be a subset of $\mathbb{R}^{d}$. For all $\eta>0$, let

$$
\mathcal{H}_{\eta}^{\xi}(S)=\inf _{\left\{C_{i}\right\}_{i \in \mathcal{I}}: S \subset \bigcup_{i \in \mathcal{I}} C_{i}} \sum_{i \in \mathcal{I}} \xi\left(\left|C_{i}\right|\right), \text { (the family of sets }\left\{C_{i}\right\}_{i \in \mathcal{I}} \text { covers } S \text { ) }
$$

where the infimum is taken over all countable families $\left\{C_{i}\right\}_{i \in \mathcal{I}}$ such that $\forall i \in \mathcal{I},\left|C_{i}\right| \leq \eta$. As $\eta$ decreases to $0, \mathcal{H}_{\eta}^{\xi}(S)$ is non-decreasing, and $\mathcal{H}^{\xi}(S)=\lim _{\eta \rightarrow 0} \mathcal{H}_{\eta}^{\xi}(S)$ defines a Borel measure on $\mathbb{R}^{d}$, called Hausdorff $\xi$-measure.

Defining the family $\xi_{\alpha}(x)=|x|^{\alpha}(\alpha \geq 0)$, there exists a unique real number $0 \leq D \leq d$ such that $D=\sup \left\{\alpha \geq 0: \mathcal{H}^{\xi_{\alpha}}(S)=+\infty\right\}=\inf \left\{\alpha: \mathcal{H}^{\xi_{\alpha}}(S)=0\right\}$ (with the convention $\sup \emptyset=0$ ). This number $D$ is called the Hausdorff dimension of $S$ and denoted $\operatorname{dim} S$. We refer the reader to $[\mathbf{1 8}, \mathbf{2 7}]$ for more details.

Let us recall the mass distribution principle [18], which allows to find lower bounds for the Hausdorff dimensions of sets. 
Lemma 2.1. Let $F$ be a Borel set in $\mathbb{R}$, and $\mu$ a Borel probability measure on $F$. Suppose that, for some $\eta>0$, there are $\alpha>0$ and a gauge function $\xi$ such that $\liminf _{x \rightarrow 0^{+}} \frac{\xi(x)}{\xi_{\alpha}(x)}>0$ and for every set $U$ with $|U| \leq \eta, \quad \mu(U) \leq C \xi(|U|)$.

Then $\mathcal{H}^{\xi}(F) \geq \mu(F) / C$ and $\operatorname{dim} F \geq \alpha$.

2.2. Scaling properties of multinomial measures and connections with b-adic expansions

Let $b$ be an integer $\geq 2$. For every $j \geq 0, \forall k \in\left\{0,1, \ldots, b^{j}-1\right\}, I_{j, k}^{b}$ denotes the $b$-adic interval $\left[k b^{-j},(k+1) b^{-j}\right)$. Then for all $x \in[0,1), I_{j}^{b}(x)$ stands for the unique $b$-adic interval of generation $j$ containing $x$, and $k_{j, x}^{b}$ is the unique integer such that $I_{j}^{b}(x)=I_{j, k_{j, x}^{b}}^{b}$.

Let us recall the definition of a multinomial measure $\mu_{\tilde{\pi}}$ on $[0,1]$.

Let $\widetilde{\pi}=\left(\pi_{0}, \pi_{1}, \ldots, \pi_{b-1}\right) \in(0,1)^{b}$ be such that $\sum_{i=0}^{b-1} \pi_{i}=1$. The measure $\mu_{\tilde{\pi}}$ is the unique probability measure on $\mathbb{R}$ satisfying the equation

$$
\mu_{\tilde{\pi}}=\sum_{i=0}^{b-1} \pi_{i} \cdot \mu_{\tilde{\pi}} \circ S_{i}^{-1}
$$

where $S_{i}$ is the mapping $S_{i}(x)=\frac{x+i}{b}[\mathbf{2 1}]$. The support of the measure $\mu_{\tilde{\pi}}$ is the whole interval $[0,1]$, and from (2.1) $\mu_{\tilde{\pi}}$ enjoys the following self-similarity property:

$$
\text { For every } b \text {-adic interval } L \text { of }[0,1), \mu_{\widetilde{\pi} \mid L}=\mu(L) \cdot \mu_{\widetilde{\pi}} \circ f_{L}^{-1},
$$

where $f_{L}$ denotes the canonical increasing affine mapping from $[0,1)$ onto $L$. The relationship between $A(\widetilde{\pi})$ and the measure $\mu_{\tilde{\pi}}$ is well-known. Indeed, as a consequence of the law of large numbers, $\mu_{\widetilde{\pi}}(A(\widetilde{\pi}))=1$. This explains the lower bound (1.4).

The next proposition, proved in Section 7, is useful for proving Theorem 1.6. It is a refinement of the fact that $\mu_{\widetilde{\pi}}(A(\widetilde{\pi}))=1$ and is based the law of the iterated logarithm.

Proposition 2.2. Let $b \geq 2$ be an integer and $\widetilde{\pi}=\left(\pi_{0}, \ldots, \pi_{b-1}\right)$ be a positive probability vector. Let $\psi:[0,1 / 2) \rightarrow \mathbb{R}^{+}$be the mapping

$$
\psi(0)=0 \text { and } \quad \psi: r \mapsto \sqrt{\frac{\log \log |\log (r)|}{|\log r|}} \text { if } r>0 .
$$

The property $\mathcal{P}_{M, \tilde{\pi}}(I)$ is said to hold for an interval I and a constant $M$ when

$$
\begin{array}{r}
M^{-1}|I|^{\alpha(\widetilde{\pi})+\psi(|I|)} \leq \mu_{\widetilde{\pi}}(I) \leq M|I|^{\alpha(\widetilde{\pi})-\psi(|I|)} \\
\text { and } \quad \forall i \in\{0,1, \ldots, b-1\}, \sup _{x \in I}\left|\phi_{i,\left[-\log _{b}|I|\right]}(x)-\pi_{i}\right| \leq M \psi(|I|) .
\end{array}
$$

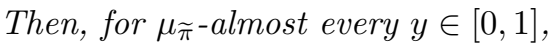

$$
\exists j(y), \forall j \geq j(y), \forall k \text { such that }\left|k-k_{j, y}^{b}\right| \leq 1, \mathcal{P}_{1, \widetilde{\pi}}\left(I_{j, k}^{b}\right) \text { holds, }
$$

Remark 2.3. Due to the definition of $\mu_{\tilde{\pi}}$, properties (2.4) and (2.5) are quite redundant, and when $I$ is a b-adic interval and $M=1$, (2.5) implies (2.4). We kept these two conditions together because, as we already said, the detection of points with digit frequencies given by $\widetilde{\pi}$ is closely related to the local behavior of $\mu_{\tilde{\pi}}$.

REMARK 2.4. The function $\psi$ satisfies the following properties:

(i) $\lim _{r \rightarrow 0^{+}} r^{-\psi(r)}=+\infty$ and $r \mapsto r^{-\psi(r)}$ is non-increasing near $0^{+}$,

(ii) $\psi(0)=0$ and $\forall \varepsilon>0, r \mapsto r^{\varepsilon-\psi(r)}$ is non-decreasing near $0^{+}$,

(iii) $\forall a>1, \exists C>0, \sup _{x \in(0,1 / 2)} \frac{\psi(x)}{\psi(x / a)} \leq C$. 
3. Upper bound for the dimensions: Part (i) of Theorem 1.6

In the sequel, with each pair $\left(x_{n}, l_{n}\right)$ and every $\delta \geq 1$ are associated the open balls

$$
I_{n}=B\left(x_{n}, l_{n}\right) \quad \text { and } \quad I_{n}^{(\delta)}=B\left(x_{n},\left(l_{n}\right)^{\delta}\right)=\left(x_{n}-l_{n}^{\delta}, x_{n}+l_{n}^{\delta}\right) .
$$

Recall a standard large deviations property for multinomial measures [7].

LEMMA 3.1. For every $\beta>0$, there is $\gamma>0$ and an integer $J_{\beta}$ such that for every $j \geq J_{\beta}$, the cardinality of the set $\mathcal{D}(j, \alpha(\widetilde{\pi}), \gamma)$ of dyadic intervals $I \subset[0,1]$ of generation $j$ such that $2^{-j(\alpha(\widetilde{\pi})+\gamma)} \leq \mu_{\widetilde{\pi}}(I)$ is less than $2^{j(\alpha(\widetilde{\pi})+\beta)}$.

We prove the upper bound for one set $\mathcal{S}(\widetilde{X}, \widetilde{\pi}, \delta, \widetilde{\varepsilon})$. Then, the upper bound for the intersection is immediate.

Since $\operatorname{dim} \mathcal{S}(\widetilde{X}, \widetilde{\pi}, \delta, \widetilde{\varepsilon})=\sup _{p \in \mathbb{Z}} \operatorname{dim}(\mathcal{S}(\widetilde{X}, \widetilde{\pi}, \delta, \widetilde{\varepsilon}) \cap[p, p+1])$, we can restrict ourselves to the case where $\left(x_{n}\right)_{n \geq 1}$ is a dense sequence in $[0,1]$ and $l_{n}$ is non-decreasing and tends to 0 at $\infty$ in the definition of $\mathcal{S}(\widetilde{X}, \widetilde{\pi}, \delta, \widetilde{\varepsilon})$.

Let us fix $D>\alpha(\widetilde{\pi}) / \delta$ and $\eta>0$. For $N \geq 1$, let us introduce the set

$$
S_{N}=\bigcup_{j \geq j_{N}} \bigcup_{n \in T_{j}:\left|I_{n}\right|^{\alpha(\widetilde{\pi})+\eta} \leq \mu_{\tilde{\pi}}\left(I_{n}\right)}\left|I_{n}\right|^{(\delta)}
$$

and the integer $j_{N}=\inf _{n \geq N}\left|\log _{2}\left(l_{n}\right)\right|$. Notice that $\mathcal{S}(\widetilde{X}, \widetilde{\pi}, \delta, \widetilde{\varepsilon}) \subset S_{N}$, hence we are going to use $S_{N}$ as a covering of $\mathcal{S}(\widetilde{X}, \widetilde{\pi}, \delta, \widetilde{\varepsilon})$.

Every interval $I_{n}^{(\delta)}$ appearing in $S_{N}$ has a length smaller than $2^{-j_{N} \delta}$. Consequently,

$$
\mathcal{H}_{2^{-j_{N} \delta}}^{\xi_{D}}(\mathcal{S}(\tilde{X}, \tilde{\pi}, \delta, \widetilde{\varepsilon})) \leq \sum_{j \geq j_{N}} \sum_{n \in T_{j}:\left|I_{n}\right|^{\alpha(\widetilde{\pi})+\eta} \leq \mu_{\tilde{\pi}}\left(I_{n}\right)}\left|I_{n}^{(\delta)}\right|^{D}
$$

We fix $\beta>0$ and $\gamma>0$ as in Lemma $3 \cdot 1$, so that $\# \mathcal{D}(j, \alpha(\widetilde{\pi}), \gamma) \leq 2^{j(\alpha(\widetilde{\pi})+\beta)}$ for $j \geq J_{\beta}$. Then, we choose then $\eta$ and $J_{\beta}$ so that $2^{-j(\alpha(\widetilde{\pi})+\eta)} /\left(2^{2 \alpha(\widetilde{\pi})+2 \eta+1}\right) \geq 2^{-(j-1)(\alpha(\widetilde{\pi})+\gamma)}$ for $j \geq J_{\beta}$. Finally, we choose $N$ large enough so that $j_{N} \geq J_{\beta}$.

We can now use the WRA property of $\widetilde{X}$, in Definition $1 \cdot 5$. For $j \geq j_{N}, 1 \leq k \leq N_{j}$ and $n \in T_{j, k}$, the interval $I_{n}$ intersects at least one dyadic interval $I$ of length $2^{-(j-1)}$ such that $\mu_{\widetilde{\pi}}(I) \geq \mu\left(I_{n}\right) / 2 \geq\left|I_{n}\right|^{\alpha(\widetilde{\pi})+\eta} / 2 \geq|I|^{\alpha(\widetilde{\pi})+\eta} / 2^{2 \alpha(\widetilde{\pi})+2 \eta+1} \geq 2^{-(j-1)(\alpha(\widetilde{\pi})+\gamma)}$. Moreover, since the $I_{m}, m \in T_{j, k}$, are pairwise distinct, an interval $I$ selected as above intersects at most two such intervals $I_{m}$. Thus, $\#\left\{n \in T_{j, k}:\left|I_{n}\right|^{\alpha(\widetilde{\pi})+\eta} \leq \mu_{\widetilde{\pi}}\left(I_{n}\right)\right\} \leq$ $2 \cdot \# \mathcal{D}(j-1, \alpha(\widetilde{\pi}), \gamma)$.

Using that $\left|I_{n}^{(\delta)}\right| \leq 2^{-(j-1) \delta}$ when $n \in T_{j, k}$, the previous remarks yield

$$
\begin{aligned}
\mathcal{H}_{2^{-j_{N} \delta}}^{\xi_{D}}(\mathcal{S}(\widetilde{X}, \widetilde{\pi}, \delta, \widetilde{\varepsilon})) & \leq \sum_{j \geq j_{N}} \sum_{k=1}^{N_{j}} \sum_{n \in T_{j, k}:\left|I_{n}\right|^{\alpha(\tilde{\pi})+\eta} \leq \mu_{\tilde{\pi}}\left(I_{n}\right)}\left|I_{n}^{(\delta)}\right|^{D} \\
& \leq \sum_{j \geq j_{N}} 2 \cdot N_{j} \cdot 2^{(j-1)(\alpha(\widetilde{\pi})+\varepsilon)} \cdot 2^{-(j-1) D \delta} .
\end{aligned}
$$

Since $N_{j}=2^{o(j)}$, we conclude that $\lim _{N \rightarrow \infty} \mathcal{H}_{2^{-j_{N}}}^{\xi_{D}}(\mathcal{S}(\tilde{X}, \tilde{\pi}, \delta, \widetilde{\varepsilon}))=0$ if $\beta$ is small enough. This implies that $\operatorname{dim} \mathcal{S}(\tilde{X}, \widetilde{\pi}, \delta, \widetilde{\varepsilon}) \leq D$, for every $D>\alpha(\widetilde{\pi}) / \delta$, hence the result. 


\section{Lower bound for the dimension: Part (ii) of Theorem $1 \cdot 6$}

Without loss of generality we restrict our study to $[0,1]$ and suppose that in the definition of the limsup sets we are concerned with, the sequence $\left(x_{n}\right)_{n \geq 1}$ is dense in $[0,1]$ and the sequence $\left(l_{n}\right)_{n \geq 1}$ is non-decreasing and converges to 0 .

Let us introduce the sequence $\left(m_{p}\right)_{p \geq 1}$ defined as follows: for every integer $q \geq 1$, for every integer $p \in\left\{\frac{q(q+1)}{2}, \ldots, \frac{(q+1)(q+2)}{2}-1\right\}, m_{p}=p-\frac{q(q+1)}{2}+1$. This sequence takes the values $1,2,1,2,3,1,2,3,4,1, \ldots$ Hence every positive integer is reached an infinite number of times by the sequence $\left(m_{p}\right)_{p \geq 1}$. Then, instead of the sequence of parameters $\left(b_{p}, \tilde{\pi}^{p}, \delta_{p}\right)_{p \geq 1}$, we consider the sequence $\left(b_{m_{p}}, \widetilde{\pi}^{m_{p}}, \delta_{m_{p}}\right)_{p \geq 1}$. By abuse of notations, this second (redundant) sequence will still be denoted $\left(b_{p}, \widetilde{\pi}^{p}, \delta_{p}\right)_{p \geq 1}$.

\subsection{Preliminary results}

Take $\psi$ as in Proposition $2 \cdot 2$. The following property is useful

$$
\exists C>1, \forall 0<r \leq s \leq 1, s^{-\psi(s)} \leq C r^{-\psi(r)} .
$$

LEMMA $4 \cdot 1$. Let $y \in[0,1], b \geq 2$ and $\widetilde{\pi}$ a probability vector in $(0,1)^{b}$. Assume that there is $j_{y} \in \mathbb{N}$ such that (2.6) holds true for $y$ and every $j \geq j_{y}$ when applied to $\mu_{\tilde{\pi}}$.

There exists a constant $M_{b}$ independent of $y$ but depending on $b$ with the following property: for every $n$ such that $y \in B\left(x_{n}, l_{n} / 2\right)$ and $\log _{b} l_{n}^{-1} \geq j_{y}+4, \mathcal{P}_{M_{b}, \tilde{\pi}}\left(I_{n}\right)$ holds.

Proof. Assume that $y \in B\left(x_{n}, l_{n} / 2\right)$ with $l_{n} \leq b^{-j_{y}-4}$. Let $j_{0}$ be the smallest integer $j$ such that $b^{-j} \leq l_{n} / 2$, and $j_{1}$ the largest integer $j$ such that $b^{-j} \geq 2 l_{n}$. We have $j_{0} \geq-\log _{b} l_{n} \geq j_{1} \geq j_{y}$. We thus ensured by construction that $j_{0}-4 \leq-\log _{b} l_{n} \leq j_{1}+4$.

We have $I_{j_{0}}^{b}(y) \subset I_{n} \subset \bigcup_{\left|k-k_{j_{1}, y}^{b}\right| \leq 1} I_{j_{1}, k}^{b}$ (see Section $2 \cdot 2$ for notations). This gives $\mu_{\tilde{\pi}}\left(I_{j_{0}}^{b}(y)\right) \leq \mu\left(I_{n}\right) \leq \sum_{\left|k-k_{j_{1}, y}^{b}\right| \leq 1} \mu_{\widetilde{\pi}}\left(I_{j_{1}, k}^{b}\right)$. Then, (2.6) and (2.4) yield $\left|b^{-j_{0}}\right|^{\alpha(\widetilde{\pi})+\psi\left(\left|b^{-j_{0}}\right|\right)} \leq$ $\mu\left(I_{n}\right) \leq 3\left|b^{-j_{1}}\right|^{\alpha(\widetilde{\pi})-\psi\left(\left|b^{-j_{1}}\right|\right)}$. Combining the fact that $j_{0}-4 \leq-\log _{b} l_{n} \leq j_{1}+4$ with (4.1) and (1.4), we obtain

$$
M_{b}{ }^{-1}\left|I_{n}\right|^{\alpha(\widetilde{\pi})+\psi\left(\left|I_{n}\right|\right)} \leq \mu\left(I_{n}\right) \leq M_{b}\left|I_{n}\right|^{\alpha(\widetilde{\pi})-\psi\left(\left|I_{n}\right|\right)}
$$

for some constant $M_{b}$ that does not depend on $y$.

Let us show that there exists $M_{b}$ such that property (2.5) holds true with $I_{n}$. First notice that since $I_{n} \subset \bigcup_{\left|k-k_{j_{1}, y}^{b}\right| \leq 1} I_{j_{1}, k}^{b}$, and since (2.5) holds with $b$-adic intervals, then $\left|\phi_{i, j_{1}}(x)-\pi_{i}\right| \leq \psi\left(2^{-j_{1}}\right)$ for all $x \in I_{n}$ and $i \in\{0, \ldots, b-1\}$. Using now Remark $2 \cdot 4$ and the monotonicity of $\psi$, since $b^{-j_{1}} / l_{n}$ is bounded independently of $y$, the constant $M_{b}$ can be chosen such that we also have $\left|\phi_{i,\left[-\log _{b} l_{n}\right]}(x)-\pi_{i}\right| \leq M_{b} \psi\left(\left|I_{n}\right|\right)$ for all $x \in I_{n}$ and $i \in\{0, \ldots, b-1\}$. As a consequence, $\mathcal{P}_{M_{b}, \widetilde{\pi}}\left(I_{n}\right)$ holds true.

Next Lemma $4 \cdot 2$ follows directly from Proposition $2 \cdot 2$.

Lemma $4 \cdot 2$. Let $\psi$ be as in (2.3). For every $j \geq 1$, we introduce the sets

$$
E(j, \widetilde{\pi})=\left\{x \in[0,1]: \forall j^{\prime} \geq j, \forall k \text { such that }\left|k-k_{j^{\prime}, y}^{b}\right| \leq 1, \mathcal{P}_{1, \widetilde{\pi}}\left(I_{j^{\prime}, k}^{b}\right) \text { holds }\right\} .
$$

These sets $(E(j, \tilde{\pi}))_{j \geq 1}$ form a non-decreasing sequence in $[0,1]$, and since (2.6) holds true for $\mu_{\tilde{\pi}}$-almost every $y \in[0,1]$, their union $\bigcup_{j \geq 1} E(j, \tilde{\pi})=E(\widetilde{\pi})$ is of full $\mu_{\tilde{\pi}}$-measure. Therefore there is an integer $\widetilde{j}(\widetilde{\pi})$ such that

$$
\widetilde{j}(\widetilde{\pi})=\inf \left\{j \geq 1: \mu_{\tilde{\pi}}(E(j, \tilde{\pi})) \geq 1 / 2\right\} .
$$


The integer $\widetilde{j}(\widetilde{\pi})$ plays an important role in the following. It heuristically indicates the scale from which the mono-dimensional behavior of $\mu_{\widetilde{\pi}}$ becomes tractable on the intervals of generation greater than $\widetilde{j}(\widetilde{\pi})$.

\subsection{Proof of part (ii) of Theorem $1 \cdot 6$}

We simplify the notations as follows: For every $p \geq 1$, we set $\mu_{p}=\mu_{\widetilde{\pi}^{p}}, \alpha_{p}=\alpha\left(\widetilde{\pi}^{p}\right)$, $\mathcal{P}_{p}=\mathcal{P}_{M_{b_{p}}, \tilde{\pi}^{p}}$ (where $M_{b_{p}}$ comes from Lemma $\left.3 \cdot 1\right), E_{p}=E\left(\widetilde{\pi}^{p}\right)$ and finally $\widetilde{j}(p)=\widetilde{j}\left(\widetilde{\pi}^{p}\right)$. We also fix for every $p \geq 1$ a sequence $\widetilde{\varepsilon}^{p}$ tending to 0 at $\infty$ such that for $n$ large enough for every $p \geq 1, \varepsilon_{n}^{p}$ is chosen so that we have in (4.2)

$$
\begin{gathered}
\left(l_{n}\right)^{\alpha_{p}+\varepsilon_{n}^{p}} \leq b_{p}{ }^{-1}\left|2 l_{n}\right|^{\alpha_{p}+\psi\left(2 l_{n}\right)} \leq \mu\left(I_{n}\right) \leq M_{b_{p}}\left|2 l_{n}\right|^{\alpha_{p}-\psi\left(2 l_{n}\right)} \leq\left(l_{n}\right)^{\alpha_{p}-\varepsilon_{n}^{p}} \\
\text { and } \forall i \in\left\{0,1, \ldots, b_{p}-1\right\},\left|\phi_{i,\left[-\log _{b_{p}} l_{n}\right]}\left(x_{n}\right)-\pi_{i}\right| \leq M_{b_{p}} \psi\left(l_{n}\right) \leq \varepsilon_{n}^{p}
\end{gathered}
$$

If $\psi$ is the function (2.3), then Proposition $2 \cdot 2$ clearly implies that $\widetilde{\varepsilon}^{p}$ can be chosen equal to $\kappa\left(l_{n}\right) \psi\left(l_{n}\right)$, where $\kappa(r)=o(\sqrt{\log \log |\log (r)|})$ and $\lim _{r \rightarrow 0} \kappa(r)=\infty$ as $r$ tends to 0 . The function $\kappa$ can even be taken constant when the set $\left\{\left(b_{p}, \widetilde{\pi}^{p}\right): p \geq 1\right\}$ is finite.

In the rest of the paper, we assume that

$$
\alpha(\widetilde{\Delta}, \widetilde{\Pi})=\inf _{p \geq 1} \frac{\alpha_{p}}{\delta_{p}}>0 .
$$

We are going to construct a generalized Cantor set $K$ included in the intersection $\mathcal{A}=\mathcal{A}(\widetilde{X}, \widetilde{\Delta}, \widetilde{\Pi}, \widetilde{E})=\bigcap_{p \geq 1} \mathcal{S}\left(\widetilde{X}, \widetilde{\pi}^{p}, \delta_{p}, \widetilde{\varepsilon}^{p}\right)$ and simultaneously a measure $m$ whose support is included in $K$, such that $m$ satisfies the monofractality condition:

$$
\forall \varepsilon>0, \forall x \in[0,1], \forall r>0 \text { small enough, } m(B(x, r)) \leq r^{\alpha(\widetilde{\Delta}, \widetilde{\Pi})-\varepsilon} .
$$

Then, applying the mass distribution principle (Lemma $2 \cdot 1$ ), the support of $m$ has a Hausdorff dimension greater than $\alpha(\widetilde{\Delta}, \widetilde{\Pi})$. Since $\mathcal{A}$ contains this support, part (iii) of Theorem 1.6 will be proved.

When $\alpha(\widetilde{\Delta}, \widetilde{\Pi})=0$, the following proof establishes that $\mathcal{A} \neq \emptyset$ but no tractable scaling property of the measure built on the Cantor set can be obtained. By part (i) of Theorem $1 \cdot 6$, we obtain $\operatorname{dim} \mathcal{A}=0$.

\subsection{Construction of the Cantor set $K$}

- First step of the construction: Let $L_{1}=[0,1]$, and consider $b_{1}, b_{2}, \mu_{1}, \delta_{1}$ and $M_{1}$. Combining (1.8) and Corollary $4 \cdot 2$, there exist an integer $\widetilde{j}(1)\left(=\widetilde{j}\left(\widetilde{\pi}^{1}\right)\right)$ and a set $E_{L_{1}}^{1}=E\left(\widetilde{j}(1), \widetilde{\pi}^{1}\right) \subset L_{1}$ such that $\mu_{1}\left(E_{L_{1}}^{1}\right) \geq 1 / 2$ and $\forall y \in E_{L_{1}}^{1}$ :

- $y \in \bigcap_{N \geq 1} \bigcup_{n \geq N} B\left(x_{n}, l_{n} / 2\right)$,

- for every $j \geq \widetilde{j}_{L_{1}},(2.6)$ (and thus (2.4) and (2.5)) holds true for $y$ and $\widetilde{\pi}^{1}$.

Our construction requires Besicovitch's covering Theorem.

THEOREM 4.3. (Theorem 2.7 of $[\mathbf{2 7}]$ ) Let $d \in \mathbb{N}^{*}$. There is a constant $Q(d)$ depending only on $d$ with the following properties. Let $T$ be a bounded subset of $\mathbb{R}^{d}$ and $\mathcal{F}$ a family of closed balls such that each point of $T$ is the center of some ball of $\mathcal{F}$. There are families $\mathcal{F}_{1}, \ldots, \mathcal{F}_{Q(d)} \subset \mathcal{F}$ covering $T$ such that each $\mathcal{F}_{i}$ is composed of disjoint balls, i.e.

$$
T \subset \bigcup_{i=1}^{Q(d)} \bigcup_{F \in \mathcal{F}_{i}} F \quad \text { and } \quad \forall F, F^{\prime} \in \mathcal{F}_{i} \text { with } F \neq F^{\prime}, F \cap F^{\prime}=\emptyset .
$$


We apply Theorem 4.3 in dimension $d=1$ with $T=E_{L_{1}}^{1}$ and with several families $\mathcal{F}_{1}(j)$ of balls constructed as follows. For any $j \geq 1$ and $y \in L_{1}$, consider the set of balls

$$
\mathbf{B}_{j}^{1}(y)=\left\{I_{n}=B\left(x_{n}, l_{n}\right): y \in B\left(x_{n}, l_{n} / 2\right) \text { and } l_{n} \in\left(\left(b_{1}\right)^{-(j+1)},\left(b_{1}\right)^{-j}\right]\right\} .
$$

Since $y \in E_{L_{1}}^{1}$, there is an infinite number of integers $j$ such that $\mathbf{B}_{j}^{1}(y) \neq \emptyset$. For such a $j$, take $I_{n} \in \mathbf{B}_{j}^{1}(y)$. Then the associated contracted interval $I_{n}^{\left(\delta_{1}\right)}$ contains an infinite number of $b_{2}$-adic intervals. Among them, let $B_{n}^{1, \delta_{1}}(y)$ be the $b_{2}$-adic interval of maximal length containing $y$ and whose closure is also contained in $I_{n}^{\left(\delta_{1}\right)}$, and define

$$
\mathbf{B}_{j}^{1, \delta_{1}}(y)=\left\{B_{n}^{1, \delta_{1}}(y): B_{n}^{1, \delta_{1}}(y) \text { is associated with } I_{n} \in \mathbf{B}_{j}^{1}(y)\right\} .
$$

Still for $y \in E_{L_{1}}^{1}$ and $j \geq \widetilde{j}(1)$, we now consider the integer

$$
n_{j, y}^{1}=\inf \left\{n: l_{n} \leq\left(b_{1}\right)^{-j} \text { and } \exists j^{\prime} \geq j \text { such that } I_{n} \in \mathbf{B}_{j^{\prime}}^{1}(y)\right\}
$$

and we introduce for every $j \geq \widetilde{j}(1)+4$ the set of balls

$$
\mathcal{F}_{1}(j)=\left\{B\left(y, 4 l_{n_{j, y}^{1}}\right): y \in E_{L_{1}}^{1}\right\} .
$$

The family $\mathcal{F}_{1}(j)$ fulfills the conditions of Theorem $4 \cdot 3$. Thus, for every $j \geq \widetilde{j}(1)+4$, $Q(1)$ families of disjoint balls $\mathcal{F}_{1}^{1}(j), \ldots, \mathcal{F}_{1}^{Q(1)}(j)$, can be extracted from $\mathcal{F}_{1}(j)$, such that their union still covers $E_{L_{1}}^{1}$. Therefore, since $\mu_{1}\left(E_{L_{1}}^{1}\right) \geq 1 / 2$, at least one the families $\mathcal{F}_{1}^{i}(j)$ verifies $\mu_{1}\left(\bigcup_{B \in \mathcal{F}_{1}^{i}(j)} B\right) \geq 1 /(2 Q(1))$. Consider one of them, say $\mathcal{F}_{1}^{i}(j)$. Again, we extract from $\mathcal{F}_{1}^{i}(j)$ a finite family of pairwise disjoint balls $\widetilde{G}_{1}(j)=\left\{B_{1}, B_{2}, \ldots, B_{N}\right\}$ such that

$$
\mu_{1}\left(\bigcup_{B_{k} \in \widetilde{G}_{1}(j)} B_{k}\right) \geq \frac{1}{4 Q(1)} .
$$

With each $B_{k}$ can be associated a point $y_{k} \in E_{L_{1}}^{1}$ so that $B_{k}=B\left(y_{k}, 4 l_{n_{j, y_{k}}^{1}}\right)$. Moreover, still by construction (see (4.5)), $I_{n_{j, y_{k}}^{1}}=B\left(x_{n_{j, y_{k}}^{1}}, l_{n_{j, y_{k}}^{1}}\right) \subset B\left(y_{k}, 4 l_{n_{j, y_{k}}^{1}}\right)=B_{k}$. Thus the contracted interval $I_{n_{j, y_{k}}^{1}}^{\left(\delta_{1}\right)}$ is also included in $B_{k}$. Finally, using Lemma $4 \cdot 1$, $\mathcal{P}_{1}\left(I_{n_{j, y_{k}}^{1}}\right)$ holds true.

By construction, for every $k \in\{1,2, \ldots, N\}, \mathbf{B}_{j}^{1, \delta_{1}}\left(y_{k}\right)$ is not empty. Let $F_{k}$ be the closure of one of the $b_{2}$-adic intervals of $\mathbf{B}_{j}^{1, \delta_{1}}\left(y_{k}\right)$. By construction we have $\left|F_{k}\right| \leq$ $\left|I_{n_{j, y_{k}}}^{\left(\delta_{1}\right)}\right| \leq C_{1}\left|F_{k}\right|$ for some constant $C_{1}$ which depends only on $b_{1}$ and $b_{2}$.

When $k$ (and thus $B_{k}$ ) is given in $\{1,2, \ldots, N\}$, we denote its associated subinterval $F_{k}$ by $\underline{B_{k}}=F_{k}$. Conversely, when a $b_{2}$-adic interval $F$ can be written $\underline{B}$ for some larger ball $B$, we write $B=\bar{F}$. Therefore, for every closed interval $F$ constructed above, we ensured

$$
C_{1}^{\prime-1}|F| \leq|\bar{F}|^{\delta_{1}} \leq C_{1}^{\prime}|F|
$$

where $C_{1}^{\prime}$ depends only on $\delta_{1}, b_{1}$ and $b_{2}$. We eventually set

$$
G_{1}(j)=\left\{\underline{B_{k}}: B_{k} \in \widetilde{G}_{1}(j)\right\} .
$$

Remark that, again by construction, if $F_{1}$ and $F_{2}$ are two distinct elements of $G_{1}(j)$, then their distance is at least $\max _{i \in\{1,2\}}\left|\overline{F_{i}}\right| / 2$ (this is due to the choice of the constant 4 in (4.6)). 
On the algebra generated by the elements of $G_{1}(j)$, the probability measure $m$ is defined by

$$
m(F)=\frac{\mu_{1}(\bar{F})}{\sum_{F_{k} \in G_{1}(j)} \mu_{1}\left(\overline{F_{k}}\right)} .
$$

Let $F \in G_{1}(j)$. Using $\mathcal{P}_{1}\left(I_{n_{j, y_{k}}^{1}}\right)$ (more precisely (4.2)), (4.8) and (4.1), we obtain

$$
\mu_{1}(\bar{F}) \leq M_{1}|\bar{F}|^{\alpha_{1}-\psi(|\bar{F}|)} \leq M_{1}\left(C_{1}^{\prime}\right)^{1 / \delta_{1}}|F|^{\alpha_{1} / \delta_{1}}|\bar{F}|^{-\psi(|\bar{F}|)} \leq C_{1}^{\prime \prime}|F|^{\alpha_{1} / \delta_{1}}|F|^{-\psi(|F|)},
$$

where $C_{1}^{\prime \prime}$ depends only on $\delta_{1}, b_{1}$ and $b_{2}$. Moreover, by (4.7) we get

$$
\sum_{F_{k} \in G_{1}(j)} \mu_{1}\left(\overline{F_{k}}\right)=\sum_{B_{k} \in \widetilde{G}_{1}(j)} \mu_{1}\left(B_{k}\right) \geq \frac{1}{4 Q(1)} .
$$

As a consequence, $\forall F \in G_{1}(j), m(F) \leq 4 Q(1) C_{1}^{\prime \prime}|F|^{\alpha_{1} / \delta_{1}}|F|^{-\psi(|F|)}$.

By Remark 2.4, an integer $j_{1}$ can be chosen large enough so that

$$
\forall F \in G_{1}\left(j_{1}\right), \max \left(4 Q(1) C_{1}^{\prime \prime}, C_{1}^{\prime},\left(b_{2}\right)^{\widetilde{j}(2)+4}\right) \leq|F|^{-\psi(|F|)} .
$$

$C_{1}^{\prime}$ is the constant used in (4.8), $\widetilde{j}(1)$ and $\widetilde{j}(2)$ are given by Lemma $4 \cdot 2$. We choose the first generation of the construction of $K$ as the $b_{2}$-adic intervals of $G_{1}:=G_{1}\left(j_{1}\right)$. By construction,

$$
\forall F \in G_{1}, m(F) \leq|F|^{\alpha_{1} / \delta_{1}-2 \psi(|F|)} \leq|F|^{\alpha(\widetilde{\Delta}, \widetilde{\Pi})-2 \psi(|F|)} .
$$

We know that by construction, for every $F \in G_{1}$, there exists $y_{k} \in E_{L_{1}}^{1}$ such that $F \subset I_{n_{j_{1}, y_{k}}^{1}} \subset \bar{F}=B\left(y_{k}, 4 l_{n_{j_{1}, y_{k}}^{1}}\right)$.

As a consequence, for every $y \in \bigcup_{F \in G_{1}} F$, there exists an integer $n$ such that $l_{n} \leq b_{1}^{-4}$, $\left|x_{n}-y\right|<l_{n}{ }^{\delta_{1}}$, and $\mathcal{P}_{1}\left(I_{n}\right)$ holds true.

- Second step of the construction: Consider $\mu_{2}, b_{2}, b_{3}$ and $\delta_{2}$.

Let us focus on one of the $b_{2}$-adic intervals $L$ of $G_{1}$. Let $j_{L}=-\log _{b_{2}}|L|$ be the scale of the interval $L$ in basis $b_{2}$, and $f_{L}$ the canonical affine bijective mapping from $[0,1]$ to $L$.

Combining again (1.8) and Corollary $4 \cdot 2$, there is an integer $\widetilde{j}(2)$ and a set $E_{2}=$ $E\left(\widetilde{j}(2), \widetilde{\pi}^{2}\right) \subset[0,1]$ such that $\mu_{2}\left(E_{2}\right) \geq 1 / 2$ and $\forall y \in E_{2}, \forall j \geq \widetilde{j}(2),(2.6)$ holds true for $y$. Notice that $\widetilde{j}(2)$ is a scale computed in basis $b_{2}$, while $j_{1}$ and $\widetilde{j}(1)$ were related to $b_{1}$.

Let us set $E_{L}^{2}=f_{L}\left(E_{2}\right)$. Let us now use the self-similarity properties of the multinomial measure $\mu_{2}$. By construction, the restriction of $\mu_{2}$ on the $b_{2}$-adic interval $L$ is exactly a copy of $\mu_{2}$, rescaled by the factor $\mu_{2}(L)$, as expressed by the equality (2.2). Precisely, if $F \subset L$, then $\mu_{2}(F)=\mu_{2}\left(\left(f_{L}\right)^{(-1)}(F)\right) \mu_{2}(L)$.

We deduce that every $y \in E_{L}^{2}$ satisfies:

- $y \in \bigcap_{N \geq 1} \bigcup_{n \geq N} B\left(x_{n}, l_{n} / 2\right)$,

- $\forall j \geq \widetilde{j}(2)+j_{L}, \forall k$ such that $\left|k-k_{j, y}^{b_{2}}\right| \leq 1,\left(\right.$ remark that $\left.\left(b_{2}\right)^{\left(j-j_{L}\right)}=\frac{\left|I_{j, k}^{b_{2}}\right|}{|L|}\right)$

$$
\left(M_{2}\right)^{-1}\left(\frac{\left|I_{j, k}^{b_{2}}\right|}{|L|}\right)^{\alpha_{2}+\psi\left(\frac{\left|I_{j, L}^{b_{2}}\right|}{|L|}\right)} \leq \frac{\mu_{2}\left(I_{j, k}^{b_{2}}\right)}{\mu_{2}(L)} \leq M_{2}\left(\frac{\left|I_{j, k}^{b_{2}}\right|}{|L|}\right)^{\alpha_{2}-\psi\left(\frac{\left|I_{j, k}^{b_{2}}\right|}{|L|}\right)}
$$

By construction we have $\mu_{2}\left(E_{L}^{2}\right) \geq \mu_{2}(L) / 2$. 
For every $j \geq \widetilde{j}(2)+j_{L}$ and $y \in E_{L}^{2}$, let us define the set of intervals

$$
\mathbf{B}_{j}^{2}(y)=\left\{I_{n}=B\left(x_{n}, l_{n}\right): y \in B\left(x_{n}, l_{n} / 2\right) \text { and } l_{n} \in\left(\left(b_{2}\right)^{-(j+1)},\left(b_{2}\right)^{-j}\right]\right\} .
$$

As for $\mathbf{B}_{j}^{1}(y)$, if $\mathbf{B}_{j}^{2}(y)$ is not empty and $I_{n} \in \mathbf{B}_{j}^{2}(y)$, then the associated contracted interval $I_{n}^{\left(\delta_{2}\right)}$ contains an infinite number of $b_{3}$-adic boxes. Among them, let $B_{n}^{2, \delta_{2}}(y)$ be the largest one containing $y$ and whose closure is also contained in $I_{n}^{\left(\delta_{2}\right)}$. Then define

$$
\mathbf{B}_{j}^{2, \delta_{2}}(y)=\left\{B_{n}^{2, \delta_{2}}(y): B_{n}^{2, \delta_{2}}(y) \text { is associated with some } I_{n} \in \mathbf{B}_{j}^{2}(y)\right\} .
$$

For $y \in E_{L}^{2}$ and $j \geq \widetilde{j}_{L}$, let us consider the integer

$$
n_{j, y}^{2}=\inf \left\{n: l_{n} \leq\left(b_{2}\right)^{-j} \text { and } \exists j^{\prime} \geq j \text { such that } I_{n} \in \mathbf{B}_{j^{\prime}}^{2}(y)\right\} .
$$

We apply again Theorem $4 \cdot 3$ to $T=E_{L}^{2}$ and to families $\mathcal{F}_{2}(j)$ of balls constructed as above. For every $j \geq \widetilde{j}(2)+j_{L}+4, \mathcal{F}_{2}(j)=\left\{B\left(y, 4 l_{n_{j, y}^{2}}\right): y \in E_{L}^{2}\right\}$. The family $\mathcal{F}_{2}(j)$ fulfills the conditions of Theorem $4 \cdot 3$ and covers the et $E_{L}^{2}$. By Theorem $4 \cdot 3$, for every $j \geq j(2)+j_{L}+4, Q(1)$ families of pairwise disjoint boxes $\mathcal{F}_{2}^{1}(j), \ldots, \mathcal{F}_{2}^{Q(1)}(j)$, whose union covers $E_{L}^{2}$, can be extracted from $\mathcal{F}_{2}(j)$.

Since $\mu_{2}\left(E_{L}^{2}\right) \geq \mu_{2}(L) / 2$, for some integer $i$ we have $\mu_{2}\left(\bigcup_{B \in \mathcal{F}_{2}^{i}(j)} B\right) \geq \mu_{2}(L) / 2 Q(1)$. As in the first step, we extract from $\mathcal{F}_{2}^{i}(j)$ a finite family of disjoint balls $\widetilde{G}_{2}^{L}(j)=$ $\left\{B_{1}, B_{2}, \ldots, B_{N}\right\}$ such that

$$
\mu_{2}\left(\bigcup_{B_{k} \in \widetilde{G}_{2}^{L}(j)} B_{k}\right) \geq \frac{\mu_{2}(L)}{4 Q(1)} .
$$

As above, with each $B_{k}$ is associated a real number $y_{k} \in E_{L}^{2}$ so that $B_{k}=B\left(y_{k}, 4 l_{n_{j, y_{k}}}\right)$, and $I_{n_{j, y_{k}}^{2}}^{\left(\delta_{2}\right)} \subset I_{n_{j, y_{k}}^{2}} \subset B_{k}$. Again, $\mathcal{P}_{2}\left(I_{n_{j, y_{k}}^{2}}\right)$ holds true.

Let $F_{k}$ be the closure of one of the $b_{3}$-adic balls of maximal diameter included in $I_{n_{j, y_{k}}^{2}}^{\left(\delta_{2}\right)}$. We still use the notation $\underline{B_{k}}=F_{k}$ and $B_{k}=\overline{F_{k}}$. This yields the analog of (4.8)

$$
C_{2}^{\prime-1}|F| \leq|\bar{F}|^{\delta_{2}} \leq C_{2}^{\prime}|F|
$$

for another constant $C_{2}^{\prime}$ which depends on $b_{2}, b_{3}$ and $\delta_{2}$. We finally define

$$
G_{2}^{L}(j)=\left\{\underline{B_{k}}: B_{k} \in \widetilde{G}_{2}^{L}(j)\right\} .
$$

On the algebra generated by the $b_{3}$-adic elements $F$ of $G_{2}^{L}(j)$, an extension of the restriction to the ball $L$ of the measure $m$ is defined by

$$
m(F)=\frac{\mu_{2}(\bar{F})}{\sum_{F_{k} \in G_{2}^{L}(j)} \mu_{2}\left(\overline{F_{k}}\right)} m(L) .
$$

Let $F \in G_{2}^{L}(j)$. Using (4.12) and an argument as Lemma $4 \cdot 1$, we get

$$
\begin{aligned}
\mu_{2}(\bar{F})=\mu_{2}(L) \frac{\mu_{2}(\bar{F})}{\mu_{2}(L)} & \leq C_{2}^{\prime} \mu_{2}(L)\left(\frac{|\bar{F}|}{|L|}\right)^{\alpha_{2}-\psi\left(\frac{|\bar{F}|}{|L|}\right)} \leq C_{2}^{\prime} \mu_{2}(L)|F|^{\alpha_{2} / \delta_{2}}|L|^{-\alpha_{2}}\left(\frac{|\bar{F}|}{|L|}\right)^{-\psi\left(\frac{|\bar{F}|}{|L|}\right)} \\
& \leq C_{2}^{\prime} \mu_{2}(L)|F|^{\alpha_{2} / \delta_{2}}|L|^{-\alpha_{2}}|F|^{-\psi(|F|)}
\end{aligned}
$$

where (4.1) has been used and $C_{2}^{\prime}$ is a constant which depends only on $b_{2}, b_{3}$ and $\delta_{2}$. 
Moreover, by (4.14) and (4.16),

$$
\sum_{F_{k} \in G_{2}^{L}(j)} \mu_{2}\left(\overline{F_{k}}\right)=\sum_{B_{k} \in \widetilde{G}_{2}^{L}(j)} \mu_{2}\left(B_{k}\right) \geq \mu_{2}(L) / 4 Q(1) .
$$

Remember now that $m(L)$ can be bounded using (4.11). Consequently,

$$
\begin{aligned}
m(F) & \leq m(L) \frac{4 Q(1)}{\mu_{2}(L)} C_{2}^{\prime} \mu_{2}(L)|F|^{\alpha_{2} / \delta_{2}}|L|^{-\alpha_{2}}|F|^{-\psi(|F|)} \\
& \leq 4 Q(1)|L|^{\alpha(\widetilde{\Delta}, \widetilde{\Pi})-2 \psi(|L|)}|F|^{\alpha_{2} / \delta_{2}}|L|^{-\alpha_{2}}|F|^{-\psi(|F|)} \\
& \leq 4 Q(1)|L|^{\alpha(\widetilde{\Delta}, \widetilde{\Pi})-\alpha_{2}-2 \psi(|L|)}|F|^{\alpha_{2} / \delta_{2}-\psi(|F|)} \\
& \leq 4 Q(1)|L|^{\alpha(\widetilde{\Delta}, \widetilde{\Pi})-\alpha_{2}-2 \psi(|L|)}|F|^{\alpha_{\infty}-\psi(|F|)}
\end{aligned}
$$

Again, $j_{2}(L)$ can be taken large enough so that for every integer $j \geq j_{2}(L)$, for every $b_{3}$-adic interval $F \in G_{2}^{L}(j)$,

$$
\max \left(4 Q(1)|L|^{\alpha(\widetilde{\Delta}, \widetilde{\Pi})-\alpha_{2}-2 \psi(|L|)}, C_{2}^{\prime},\left(b_{3}\right)^{\widetilde{j}(3)+4}\right) \leq|F|^{-\psi(|F|)} .
$$

Then, taking $j_{2}=\max \left\{j_{2}(L): L \in G_{1}\right\}$, and defining

$$
G_{2}=\bigcup_{L \in G_{1}} G_{2}^{L}\left(j_{2}\right)
$$

formula (4.17) yields an extension of the measure $m$ to the algebra generated by the elements of $G_{1} \bigcup G_{2}$ and such that for every $F \in G_{1} \bigcup G_{2}$,

$$
m(F) \leq|F|^{\alpha(\widetilde{\Delta}, \widetilde{\Pi})-2 \psi(|F|)} .
$$

Notice that by construction, for every $F \in G_{2},|F| \leq \max _{F \in G_{1}} 2\left(\left(b_{2}\right)^{-4}|F|\right)^{\delta_{2}}$.

- Recursive construction scheme: The induction is achieved as follows. Assume that $q$ generations of closed intervals $G_{1}, \ldots, G_{q}$ are found for some integer $q \geq 2$, each generation $G_{p}$ consisting in $b_{p+1}$-adic intervals. Assume also that a probability measure $m$ on the algebra generated by $\bigcup_{1 \leq p \leq q} G_{p}$ is defined and that the following properties hold (they hold true for $p=2$ because of steps one and two of the construction):

(i) For every $1 \leq p \leq q$, the elements of $G_{p}$ are closed pairwise disjoint $b_{p+1}$-adic intervals, and for $2 \leq p \leq q, \max _{F \in G_{p}}|F| \leq 2\left(b_{p}\right)^{-4 \delta_{p}} \max _{F \in G_{p-1}}|F|^{\delta_{p}}$.

For every $1 \leq p \leq q$, with each $F \in G_{p}$ is associated an interval $\bar{F}$ such that $F \subset \bar{F}$ and $|F|^{1+\psi(|F|)} \leq|\bar{F}|^{\delta_{p}} \leq|F|^{1-\psi(|F|)}$.

The intervals $\bar{F}, F \in G_{p}$, are pairwise disjoint, and if $F_{1}$ and $F_{2}$ are two distinct elements of $G_{p}$, then their mutual distance is at least $\max _{i \in\{1,2\}}\left|\overline{F_{i}}\right| / 2$.

(ii) For every $2 \leq p \leq q$, each element $F$ of $G_{p}$ is included in an element $L$ of $G_{p-1}$. Moreover, $\bar{I} \subset L, \log _{b_{p}}\left(|\bar{F}|^{-1}\right) \geq \widetilde{j}(p)+\log _{b_{p}}\left(|L|^{-1}\right)$ and $\bar{F} \cap E_{L}^{p} \neq \emptyset$ (where $E_{L}^{p}=f_{L}\left(E_{p}\right)$ and $E_{p}$ is defined as $E_{2}$, i.e. $\left.E_{p}=E\left(\widetilde{j}(p), \widetilde{\pi}^{p}\right)\right)$.

(iii) For every $1 \leq p \leq q$, for every $F \in G_{p}$, there is an integer $n$ such that $F \subset I_{n}^{\left(\delta_{p}\right)} \subset$ $\bar{F}$ and $\mathcal{P}_{p}\left(I_{n}\right)$ holds.

(iv) For every $F \in \bigcup_{1 \leq p \leq q} G_{p}, m(F) \leq|F|^{\alpha(\widetilde{\Delta}, \widetilde{\Pi})-2 \psi(|F|)}$. 
(v) For every $2 \leq p \leq q, L \in G_{p-1}$, and $F \in G_{p}$ such that $F \subset L$,

$$
\begin{aligned}
\left(M_{p}\right)^{-1}\left(\frac{|\bar{F}|}{|L|}\right)^{\alpha_{p}+\psi\left(\frac{|\bar{F}|}{|L|}\right)} \leq \frac{\mu_{p}(\bar{F})}{\mu_{p}(L)} \leq M_{p}\left(\frac{|\bar{F}|}{|L|}\right)^{\alpha_{p}-\psi\left(\frac{|\bar{F}|}{|L|}\right)}, \\
\text { and } \quad m(F) \leq 4 Q(1) m(L) \frac{\mu_{p}(\bar{F})}{\mu_{p}(L)} .
\end{aligned}
$$

(vi) For every $1 \leq p \leq q$, for every $F \in G_{p},\left(b_{p+1}\right)^{\widetilde{j}(p+1)+4} \leq|F|^{-\psi(|F|)}$.

The construction of a generation of intervals $G_{q+1}$ and an extension of $m$ to the algebra generated by the elements of $\bigcup_{1 \leq p \leq q+1} G_{p}$ such that properties (i) to (vi) hold true for $q+1$ is achieved as in steps $q=1$ and 2 . These steps are quickly indicated:

- First let $n_{q}$ be the largest integer among the $n_{j_{q}, y_{k}}^{q}$, where $y_{k}$ is such that $\underline{B_{k}}=$ $B\left(y_{k}, 4 l_{n_{j q, y_{k}}^{q}}\right) \in G_{q}$. Consider then one of the $b_{q+1}$-adic intervals $L$ in $G_{q}$, and let $\overline{j_{L}=-\log _{b_{q+1}}}|L|$.

- Consider the integer $\widetilde{j}(q+1)$ and the set $E_{q+1}=E\left(\widetilde{j}(q+1), \widetilde{\pi}^{q}\right)$ such that $\mu_{q}\left(E_{q+1}\right) \geq 1 / 2$, and set $E_{L}^{q+1}=f_{L}\left(E_{q+1}\right)$.

For every $y \in E_{L}^{q+1}$, we have (notice that $\mu_{q+1}\left(E_{L}^{q+1}\right) \geq \mu_{q+1}(L) / 2$ )

- $y \in \bigcap_{N \geq 1} \bigcup_{n \geq N} B\left(x_{n}, l_{n} / 2\right)$,

$-\forall j \geq \widetilde{j}(q+1)+j_{L}, \forall k$ such that $\left|k-k_{j, y}^{b_{q+1}}\right| \leq 1$, (4.20) holds with $p=q+1$.

- For every $j \geq \widetilde{j}(q+1)+j_{L}$ and $y \in E_{L}^{q+1}$, the sets $\mathbf{B}_{j}^{q+1}(y)$ and $\mathbf{B}_{j}^{q+2, \delta_{q+2}}(y)$, as well as the integers $n_{j, y}^{q+1}$, are defined as when $q+1=1$ or 2 .

Theorem $4 \cdot 3$ yields a finite family of disjoint balls $\widetilde{G}_{q+1}^{L}(j)$ such that

$$
\mu_{q+1}\left(\bigcup_{B_{k} \in \widetilde{G}_{q+1}^{L}(j)} B_{k}\right) \geq \frac{\mu_{q+1}(L)}{4 Q(1)} .
$$

- Defining then $B_{k}=F_{k}$ and $B_{k}=\overline{F_{k}}$, we obtain the analog of (4.15)

$$
C_{q+1}^{\prime}{ }^{-1}|F| \leq|\bar{F}|^{\delta_{q+1}} \leq C_{q+1}^{\prime}|F|,
$$

for another constant $C_{q+1}^{\prime}$ which depends on $b_{q+1}, b_{q+2}$ and $\delta_{q+1}$. We eventually define a set of $b_{q+2}$-adic intervals

$$
G_{q+1}^{L}(j)=\left\{\underline{B_{k}}: B_{k} \in \widetilde{G}_{q+1}^{L}(j)\right\} .
$$

- On the algebra generated by the $b_{q+2}$-adic elements $F$ of $G_{q+1}^{L}(j)$, an extension of the restriction to the ball $L$ of the measure $m$ is defined by

$$
m(F)=\frac{\mu_{q+1}(\bar{F})}{\sum_{F_{k} \in G_{q+1}^{L}(j)} \mu_{q+1}\left(\overline{F_{k}}\right)} m(L) .
$$

This measure has the required scaling properties, provided that $j$ can be taken large enough: We consider the integer $j_{q+1}(L)$ so that for every integer $j \geq$ $j_{q+1}(L)$, for every $b_{q+2}$-adic interval $F \in G_{q+1}^{L}(j)$,

$$
\max \left(4 Q(1)|L|^{\alpha(\widetilde{\Delta}, \widetilde{\Pi})-\alpha_{q+1}-2 \psi(|L|)}, C_{q+1}^{\prime},\left(b_{q+2}\right)^{\widetilde{j}(q+2+4)}\right) \leq|F|^{-\psi(|F|)} .
$$

Then, taking $j_{q+1}=\max \left\{j_{q+1}(L): L \in G_{q}\right\}$, and defining

$$
G_{q+1}=\bigcup_{L \in G_{q}} G_{q+1}^{L}\left(j_{q+1}\right),
$$


formula (4.23) yields an extension of the measure $m$ to the algebra generated by the elements of $\bigcup_{p=1}^{q+1} G_{p}$ and such that for every $F \in \bigcup_{p=1}^{q+1} G_{p}$, (4.19) holds true.

- Putting everything together: By induction, and because of the separation property (i), we get a sequence $\left(G_{q}\right)_{q \geq 1}$ and a probability measure $m$ on $\sigma\left(F: F \in \bigcup_{q \geq 1} G_{q}\right)$ such that properties (i) to (vi) hold for every $q \geq 2$. We now define

$$
K=\bigcap_{q \geq 1} \bigcup_{F \in G_{q}} I
$$

The measure $m$ can be extended to $\mathcal{B}([0,1])$ by the usual way: we set $m(B):=m(B \cap$ $K)$ for $B \in \mathcal{B}([0,1])$. By construction, $m(K)=1$. Moreover, combining the redundancy introduced in the very beginning of Section 4 and property (iii), we have $K \subset$ $\bigcap_{q \geq 1} \mathcal{S}\left(\widetilde{X}, \widetilde{\pi}^{q}, \delta_{q}, \widetilde{\varepsilon}^{q}\right)=\mathcal{A}$. Finally, property (iv) implies that for every $I \in \bigcup_{q \geq 1} G_{q}$,

$$
m(F) \leq|F|^{\alpha(\widetilde{\Delta}, \widetilde{\Pi})-2 \psi(|F|)} .
$$

- Verification of the property (4.4) for $m$ : If $I \in G_{q}$, we set $g(I)=q$.

Let us fix $B$ an open interval of $[0,1]$ of length less than the one of the elements of $G_{1}$, and assume that $B \cap K \neq \emptyset$. Let $L$ be the element of largest diameter in $\bigcup_{p \geq 1} G_{p}$ such that $B$ intersects at least two elements of $G_{g(L)+1}$ included in $L$. This implies that $B$ does not intersect any other element of $G_{g(L)}$, and as a consequence $m(B) \leq m(L)$.

Let us distinguish three cases: We set $q=g(L)$.

- If $|B| \geq|L|$, then by $(4.25)$

$$
m(B) \leq m_{\delta}(L) \leq|L|^{\alpha(\widetilde{\Delta}, \widetilde{\Pi})-2 \psi(|L|)} \leq C|B|^{\alpha(\widetilde{\Delta}, \widetilde{\Pi})-2 \psi(|B|)},
$$

for some constant $C$ which depends on $\alpha(\widetilde{\Delta}, \widetilde{\Pi})$ and $\psi$.

- If $|B| \leq\left(b_{q+1}\right)^{-\widetilde{j}(q+1)-3}|L|$, let $L_{1}, \ldots, L_{r}$ be the elements of $G_{q+1}$ that intersect $B$. We use property $(\mathbf{v})$ to get

$$
m(B)=\sum_{i=1}^{r} m\left(B \cap L_{i}\right) \leq 4 Q(1) \frac{m(L)}{\mu_{q+1}(L)} \sum_{i=1}^{r} \mu_{q+1}\left(\bar{L}_{i}\right) .
$$

Let $j_{0}$ be the unique integer such that $\left(b_{q+1}\right)^{-j_{0}} \leq|B|<\left(b_{q+1}\right)^{-j_{0}+1}$ (recall that the intervals of $G_{q+1}$ are $b_{q+2}$-adic intervals). Assume that $B$ intersects for instance the intervals $L_{i_{1}}$ and $L_{i_{2}}$. Then, by (i), $|B| \geq \max \left(\left|\bar{L}_{i_{1}}\right|,\left|\bar{L}_{i_{2}}\right|\right) / 2$ when $j_{0}$ is large enough. Hence, when $|B|$ is small enough, $|B| \geq\left(\max _{i=1, \ldots, r}\left|\bar{L}_{i}\right|\right) / 2$ and the scale of the intervals $\bar{L}_{i}$ (defined as $\left.\left[-\log _{b_{q+1}}\left|\bar{L}_{i}\right|\right]\right)$ is always larger than $j_{0}-\left[\log _{b_{q+1}} 2\right] \geq j_{0}-2$.

By property (ii), for each $i \in\{1, \ldots r\}, E_{L}^{q+1} \cap \bar{L}_{i} \neq \emptyset$. Let $y \in E_{L}^{q+1} \cap \bar{L}_{i}$ for some $i$, and let us consider the $b_{q+2}$-adic interval $I_{j_{0}-2, k_{j_{0}-2, y}^{b_{q+1}}}^{b_{q+2}}$. For every $z \in \bar{L}_{i},|y-z| \leq\left(b_{q+1}\right)^{-\left(j_{0}-2\right)}$. We deduce that

$$
\bar{L}_{i} \subset \bigcup_{k: \mid k-k_{j_{0}-2, y}^{b_{q+1} \mid \leq 1}} I_{j_{0}-2, k}^{b_{q+1}} .
$$

Since $B \cap L_{i} \neq \emptyset$, the distance between $y$ and $B$ is at most $\left(b_{q+1}\right)^{-\left(j_{0}-2\right)}$. As a consequence, 
when $L_{i^{\prime}} \neq L_{i}$, the distance between $y$ and $L_{i^{\prime}}$ is lower than $\left(b_{q+1}\right)^{-\left(j_{0}-3\right)}$. This yields

$$
\bigcup_{i=1}^{r} \bar{L}_{i} \subset \bigcup_{k:\left|k-k_{j_{0}-3, y}^{b_{q}+1}\right| \leq 1} I_{j_{0}-3, k}^{b_{q+1}} .
$$

Since $y \in E_{L}^{q+1}$ and $j_{0} \geq-\log _{b_{q+1}}|L|+\widetilde{j}(q+1)+3$, (2.4) ensures the control of the $\mu_{q^{-}}$ mass of the unions of all the balls that appear on the left hand-side of (4.28) by the sum of the masses of the $3 b_{q+2}$-adic intervals $I_{j_{0}-3, k}^{b_{q+1}}$ with $\left|k-k_{j_{0}-3, y}^{b_{q+1}}\right| \leq 1$. By construction we have $\left|I_{j_{0}-3, k}^{b_{q+1}}\right| \leq b_{q+1}^{3}|L|$ so that

$$
\begin{aligned}
\mu_{q+1}\left(I_{j_{0}-3, k}^{b_{q+1}}\right) & \leq \mu_{q+1}(L)\left(\frac{\left|I_{j_{0}-3, k}^{b_{q+1}}\right|}{|L|}\right)^{\alpha(q+1)-\psi\left(\frac{\mid I_{j_{0}-3, k}^{b_{q+1} \mid}}{|L|}\right)} \\
& \leq \mu_{q+1}(L)\left(b_{q+1}^{3}\right)^{\alpha(q+1)}\left(\frac{|B|}{|L|}\right)^{\alpha(q+1)}\left(\frac{\left|I_{j_{0}-3, k}^{b_{q+1}}\right|}{|L|}\right)^{-\psi\left(\frac{\mid I_{j_{0}-3, k}^{b_{q+1} \mid}}{|L|}\right)} .
\end{aligned}
$$

Since $|B| \leq \max \left(|L|, \frac{\left|I_{j_{0}-3, k}^{b_{q}-1}\right|}{|L|}\right)$, we can use (4.1) as well as (vi) and the fact that $\alpha(q+1) \leq 1$ to obtain

$$
\left\{\begin{array}{l}
\left(b_{q+1}^{3}\right)^{\alpha(q+1)} \leq|L|^{-\psi(|L|)} \leq C|B|^{-\psi(|B|)} \\
\left(\frac{\mid I_{j_{0}-3, k}^{b_{q}+1}}{|L|}\right)^{-\psi\left(\frac{\mid I_{j_{0}-3, k}^{b_{0} \mid}}{|L|}\right)} \leq C|B|^{-\psi(|B|)}
\end{array} .\right.
$$

Consequently,

$$
\mu_{q+1}\left(I_{j_{0}-3, k}^{b_{q+1}}\right) \leq C^{2} \mu_{q+1}(L)\left(\frac{|B|}{|L|}\right)^{\alpha(q+1)}|B|^{-2 \psi(|B|)} .
$$

Using that the $\bar{L}_{i}$ are pairwise disjoint, for $|B|$ small enough, we deduce successively from $(4.27),(4.28)$ and the previous inequality that $m(B) \leq m(L) \frac{4 Q(1)}{\mu_{q+1}(L)} \mu_{q+1}\left(\bigcup_{i=1}^{r} \bar{L}_{i}\right)$, and

$$
\begin{aligned}
m(B) & \leq 3 m(L) \mu_{q+1}(L) \frac{4 Q(1)}{\mu_{q+1}(L)}\left(\frac{|B|}{|L|}\right)^{\alpha(q+1)}|B|^{-2 \psi(|B|)} \\
& \leq 12 Q(1) m(L)\left(\frac{|B|}{|L|}\right)^{\alpha(\widetilde{\Delta}, \widetilde{\Pi}) \delta_{q+1}}|B|^{-2 \psi(|B|)} .
\end{aligned}
$$

We then use (4.25): Indeed, $m(L) \leq|L|^{\alpha(\widetilde{\Delta}, \widetilde{\Pi})-2 \psi(|L|)} \leq C|L|^{\alpha(\widetilde{\Delta}, \widetilde{\Pi})}|B|^{-2 \psi(|B|)}$, which implies

$$
m(B) \leq C^{\prime}|B|^{\alpha(\widetilde{\Delta}, \widetilde{\Pi})}|B|^{-4 \psi(|B|)}\left(\frac{|B|}{|L|}\right)^{\alpha(\widetilde{\Delta}, \widetilde{\Pi})\left(\delta_{q+1}-1\right)} \leq C^{\prime}|B|^{\alpha(\widetilde{\Delta}, \widetilde{\Pi})}|B|^{-4 \psi(|B|)}
$$

with $C^{\prime}=12 Q(1) C$.

- $\left(b_{q+1}\right)^{-\tilde{j}(q+1)-3}|L|<|B| \leq|L|:$ at most $\left(b_{q+1}\right)^{\widetilde{j}(q+1)+4}$ contiguous intervals of length $\left(b_{q+1}\right)^{-\tilde{j}(q+1)-3}|L|$ are needed to cover $B$. For these intervals, (4.29) can be used. Also, 
by (vi), $\left(b_{q+1}\right)^{\widetilde{j}(q+1)+4} \leq|L|^{-\psi(L)} \leq C|B|^{-\psi(|B|)}$, hence for $|B|$ small enough

$$
\begin{aligned}
m(B) & \leq C^{\prime}\left(b_{q+1}\right)^{\widetilde{j}(q+1)+4}\left(\left(b_{q+1}\right)^{-\widetilde{j}(q+1)-3}|L|\right)^{\alpha(\widetilde{\Delta}, \widetilde{\Pi})-4 \psi\left(\left(b_{q+1}\right)^{-\tilde{j}(q+1)-3}|L|\right)} \\
& \leq C C^{\prime}|B|^{\alpha(\widetilde{\Delta}, \widetilde{\Pi})-5 \psi(|B|)} .
\end{aligned}
$$

Remembering (4.26) and (4.29), and using assumption (1), we find a constant $C$ such that for every interval $B$ of $[0,1]$ small enough, $m(B) \leq C|B|^{\alpha(\widetilde{\Delta}, \widetilde{\Pi})-5 \psi(|B|)}$. This yields (4.4).

\section{Large intersections properties. Part (iv) of Theorem $1 \cdot 6$}

Let $\tilde{X}=\left\{\left(x_{n}, l_{n}\right)\right\}_{n \geq 0}$ and $\left(b_{p}, \widetilde{\pi}^{p}, \widetilde{\varepsilon}^{p}\right)_{p \geq 1}$ be as in the statement. Let $\left(f_{k}\right)_{k \geq 1}$ be a sequence of similarity transformations on $\mathbb{R}$. Recall that we want to prove that

$$
\operatorname{dim}\left(\bigcap_{k \geq 1} f_{k}\left(\bigcap_{p \geq 1} \mathcal{S}\left(\widetilde{X}, \widetilde{\pi}^{p}, \delta_{p}, \widetilde{\varepsilon}^{p}\right)\right)\right) \geq \alpha(\widetilde{\Delta}, \widetilde{\Pi})
$$

Notice that $\bigcap_{k \geq 1} f_{k}\left(\bigcap_{p \geq 1} \mathcal{S}\left(\tilde{X}, \widetilde{\pi}^{p}, \delta_{p}, \widetilde{\varepsilon}^{p}\right)\right)=\bigcap_{k \geq 1} \bigcap_{p \geq 1} f_{k}\left(\mathcal{S}\left(\tilde{X}, \widetilde{\pi}^{p}, \delta_{p}, \widetilde{\varepsilon}^{p}\right)\right)$. For every $k \geq 1$, let $r_{k}>0$ stand for the contraction factor of $f_{k}$, and denote $f_{k}^{-1}([0,1])$ by $I_{k}$. Also, define for $k \geq 1$ and $p \geq 1$

$$
\mathcal{S}_{k}\left(\tilde{X}, \widetilde{\pi}^{p}, \delta_{p}, \widetilde{\varepsilon}^{p}\right)=\bigcap_{N \geq 0} \bigcup_{n \geq N: x_{n} \in I_{k}, \mathcal{C}\left(\widetilde{\pi}^{p}, x_{n}, l_{n}, \varepsilon_{n}^{p}\right) \text { holds }} B\left(f_{k}\left(x_{n}\right),\left(r_{k} l_{n}\right)^{\delta_{p}}\right) .
$$

It is enough to show that $\operatorname{dim} \bigcap_{k \geq 1} \bigcap_{p \geq 1} \mathcal{S}_{k}\left(\widetilde{X}, \widetilde{\pi}^{p}, \delta_{p}, \widetilde{\varepsilon}^{p}\right) \geq \alpha(\widetilde{\Delta}, \widetilde{\Pi})$.

The proof is directly inspired by the constructive proof of last Section 4 . We briefly explain how to adapt the computations of Section 4.

For every $k \geq 1$ and $n \in I_{k}$, denote the $x_{n}^{(k)}=f_{k}\left(x_{n}\right)$ and $l_{n}^{k}=r_{k} l_{n}$. Instead of having only one family $\left\{\left(x_{n}, l_{n}\right)\right\}_{n \geq 1}$, we deal now with a sequence of families $\left\{\left(x_{n}^{(k)}, l_{n}^{(k)}\right)\right\}_{n \geq 1, k \geq 1}$. We thus simplify the notations, in three steps:

- Let $\varphi: \mathbb{N}^{*} \rightarrow \mathbb{N}^{*} \times \mathbb{N}^{*}$ be a bijection. For every $q \geq 1$, when $\varphi(q)=(k, p)$, we set $b_{q}=b_{p}, \widetilde{\pi}^{q}=\widetilde{\pi}^{p}, \delta_{q}=\delta_{p}$ and finally $\widetilde{X}^{q}=\left\{\left(x_{n, q}, l_{n, q}\right)\right\}_{n \geq 0}=\left\{\left(x_{n}^{(k)}, l_{n}^{(k)}\right)\right\}_{n \geq 1}$. We thus obtain a sequence $\left(b_{q}, \tilde{\pi}^{q}, \delta_{q}, \tilde{X}^{q}\right)_{q \geq 1}$. Also, $f_{q}=f_{k}$.

- For $q \geq 1$, the elements of the image by $f_{q}$ of the $b_{q}$-adic grid (consisting in $b_{q}$-adic intervals of length smaller than or equal to 1 ) are called the $\widetilde{b}_{q}$-adic intervals.

Finally, for $n \geq 1$ and $\delta \geq 1$, the balls $B\left(x_{n, q}, l_{n, q}\right)$ and $B\left(x_{n, q}, l_{n, q}^{(\delta)}\right)$ are respectively denoted $I_{n, q}$ and $I_{n, q}^{(\delta)}$.

- We now apply the same redundancy procedure as in Section 4 (using the sequence $\left.m(p))_{p \geq 1}\right)$ : Consider the redundant sequence $\left(b_{m(q)}, \widetilde{\pi}^{m(q)}, \delta_{m(q)}, \widetilde{X}^{m(q)}\right)$, still denoted by abuse of notations $\left(b_{q}, \widetilde{\pi}^{q}, \delta_{q}, \widetilde{X}^{q}\right)$. As in Section 4 , every pair of integers $(k, p)$ (thus every $f_{k}\left(\mathcal{S}\left(\widetilde{X}, \widetilde{\pi}^{p}, \delta_{p}, \widetilde{\varepsilon}^{p}\right)\right)$ ) is considered an infinite number of times.

We now construct a Cantor set $K$ in $\bigcap_{k \geq 1} \bigcap_{p \geq 1} \mathcal{S}_{k}\left(\tilde{X}, \tilde{\pi}^{p}, \delta_{p}, \widetilde{\varepsilon}^{p}\right)$ as well as a convenient measure on $K$ by modifying the proof performed in the previous section as follows:

- First step: Without loss of generality, we suppose that $f_{1}$ is the identity function, so that the first step is identical to the one of Section 4 with the approximation family $\widetilde{X}^{1}$, except that the elements of $G_{1}$ are chosen to be $\widetilde{b}_{2}$-adic intervals. A measure $m$ with a scaling behavior as (4.11) is obtained.

- Second step: We change the approximation family and consider the family $\widetilde{X}^{2}$ 
instead of $\widetilde{X}^{1}$. We consider the family of intervals $\widetilde{G}_{1}=\left\{f_{2}^{-1}(F): F \in G_{1}\right\}$ and the measure $\widetilde{m}=m \circ f_{k}$. The elements of $\widetilde{G}_{1}$ are not, in general, subintervals of $[0,1]$.

In each interval $L$ of this family, we perform the same construction as in the second step of the proof of Theorem 1.6(ii). Notice that this procedure is feasible, since the selection of intervals (1.6) is achieved using only the fractional parts of the real numbers appearing in the approximation family we consider.

We get a second generation of intervals $\widetilde{G}_{2}^{L}$, consisting in $b_{3}$-adic intervals, and an extension of $\widetilde{m}$ to $\widetilde{G}_{2}=\bigcup_{L \in \widetilde{G}_{1}} \widetilde{G}_{2}^{L}$ so that the properties of the measure obtained in the second step of the proof of Theorem 1.6(ii) hold true for $\widetilde{m}$ and the elements of $\widetilde{G}_{1} \cup \widetilde{G}_{2}$.

Taking the images of $\widetilde{G}_{2}$ and $\widetilde{m}$ by $f_{2}$ yields a second generation $G_{2}$ made of $\widetilde{b}_{3}$ adic subintervals of $[0,1]$, as well as an extension of $m$ to these intervals so that all the properties satisfied by the similar objects in the first and second steps of the construction performed in Section 4 are also satisfied by the new intervals and measure we consider in this section. Moreover, the construction is done so that the image of each $F \in G_{2}$ is contained in an interval of the form $I_{n, 2}^{\left(\delta_{2}\right)}$ such that $f_{2}\left(x_{n}\right) \in[0,1]$ and $\mathcal{P}_{M_{b_{2}}, \widetilde{\pi}^{2}}\left(I_{n, 2}\right)$ holds true.

- Induction and definition of the Cantor set: We use the same approach as in the second step to construct by induction a sequence $\left(G_{p}\right)_{p \geq 1}$ of families of intervals such that for each $p \geq 1$ the elements of $G_{p}$ are $\widetilde{b}_{p}$-subintervals of $[0,1]$, as well as a measure $m$ on $K=\bigcap_{p \geq 1} \bigcup_{F \in G_{p}} F$ such that the properties (i) to (vi) of Section 4 are satisfied, except that in part (iii), $\mathcal{P}_{p}\left(I_{n}\right)$ and $I_{n}^{\left(\delta_{p}\right)}$ must be replaced by $\mathcal{P}_{M_{b_{p}}, \widetilde{\pi}^{p}}\left(I_{n, p}\right)$ and $I_{n, p}^{\left(\delta_{p}\right)}$, and " $F \subset I_{n}^{\left(\delta_{p}\right)} \subset \bar{F} "$ by $" F \subset f_{k}\left(I_{n}^{\left(\delta_{p}\right)}\right) \subset \bar{F} "$.

- Scaling properties of the associated measure $m$ : The verification of the fact that for all $\varepsilon>0, m(B) \leq|B|^{\alpha(\widetilde{\Delta}, \widetilde{\Pi})-\varepsilon}$ for all small enough ball, follows exactly the same lines as in Section 4. Hence the result.

The verifications are left to the reader.

\section{Examples of WRA families $\widetilde{X}=\left\{\left(x_{n}, l_{n}\right)\right\}_{n \in \mathbb{N}}$}

- The family of the b-adic numbers.

Fix $b$ an integer $\geq 2$. Let us consider the sequence $\left\{\left(k b^{-j}, 2 b^{-j}\right)\right\}$, for $j \in \mathbb{N}$ and $k \in$ $\left\{0, \ldots, b^{j}-1\right\}$. By construction, for every $j \geq 2, \bigcup_{k \in\left\{0, \ldots, b^{j}-1\right\}^{d}} B\left(k b^{-j}, b^{-j}\right)=[0,1]^{d}$. Hence (1.8) is satisfied, and the family is WRA.

- The family of the rational numbers. It is already used in (1.1) and described in Section 1. It is classical [19] that $\left\{\left(p / q, 1 / q^{2}\right)\right\}_{q \geq 1, p \wedge q=1}$ is WRA and satisfies (1.8) (except for a countable set), while the whole family $\left\{\left(p / q, 1 / q^{2}\right)\right\}_{q \geq 1, p \in\{0, \ldots, q-1\}}$, is not.

- The family of algebraic numbers of bounded degree $\geq 2$.

Recall that the height $H(x)$ of an algebraic number $x$ is the maximum of the absolute values of the relatively prime integer coefficients in the minimal polynomial for $x$, and that the degree of $x$ is the degree of the minimal polynomial for $x$.

Let $m \geq 2$. Let $\lambda$ stand for the restriction of the Lebesgue measure to $[0,1]$. The set of algebraic numbers of degree at most $m$ contained in $[0,1]$ is countable. Let us represent its elements as a sequence $\left(x_{n}\right)_{n \geq 1}$.

For $\epsilon \in(0,1)$ and $w>0$, let $\left(l_{n}(w, \epsilon)=H\left(x_{n}\right)^{-(w+1-\epsilon)}\right)_{n \geq 1}$, and let $A(w, \epsilon)=$ $\lim \sup _{n \geq 1}\left(x_{n}-l_{n}(w, \epsilon), x_{n}+l_{n}(w, \epsilon)\right)$. It follows from [5] (see also [10]) that there ex- 
ists a countable set $D$ such that for all $\varepsilon>0,(0,1) \backslash D \subset A\left(w_{m}, \epsilon\right)$, where

$$
w_{m}=\frac{m}{4}+\frac{\sqrt{m^{2}+16 m-8}}{4} .
$$

Then, Remark 1.7 (1) shows that this is enough for the conclusion of Theorem 1.6(ii) to hold with $\left\{\left(x_{n}, l_{n}\left(w_{m}, \epsilon\right)\right)\right\}_{n}$.

On the other hand, we learn from [10] that for all $\epsilon>0$ the set $A(m, \epsilon)$ is of full $\lambda$-measure. This is enough to adapt our proof of Theorem 1.6. Using $l_{n}=l_{n}(m, \epsilon)$, and taking $\widetilde{\pi}^{p}=\lambda$ and $\delta_{p}=\frac{m+1-\epsilon}{m-\epsilon}$ for every $p \geq 1$, we show that $\frac{m-\epsilon}{m+1-\epsilon} \leq \operatorname{dim} A(m+$ $1, \epsilon)$, which is finer than the lower bound $\frac{w_{m}+1-\epsilon}{m+1-\epsilon}$ obtained when using $l_{n}=l_{n}\left(w_{m}, \epsilon\right)$. Nevertheless, the approach in [1] yields $\operatorname{dim} A(m+1, \epsilon)=1$. All these remarks show that for families of algebraic numbers, our approach does not provide sharp lower bounds unless the following conjecture holds true (see [10] for instance): For all $\epsilon>0$ there exists a countable set $D$ such that $(0,1) \backslash D \subset A(m+1, \epsilon)$.

- The family $\{(z+\{n \alpha\}, 1 / n)\}_{n \geq 1, z \in \mathbb{Z}}$.

Let $\alpha$ be an irrational number. For every $n \in \mathbb{N}$, we denote by $\{n \alpha\}$ the fractional part of $n \alpha$. We have (see Theorem II.B in [11] for instance)

$$
(0,1) \backslash(\mathbb{Z}+\alpha \mathbb{Z}) \subset \bigcap_{N \geq 1} \bigcup_{n \geq N} B(\{n \alpha\}, 1 / 2 n) .
$$

Thus, the conclusion of Theorem $1 \cdot 6$ hold with the family $\{(z+\{n \alpha\}, 1 / n)\}_{n \geq 1, z \in \mathbb{Z}}$ for the results on lower bounds.

The following property concerning the redundancy holds (see [3]):

Proposition 6.1. The approximation family $\{(z+\{n \alpha\}, 1 / n)\}_{n \geq 1, z \in \mathbb{Z}}$ is WRA if and only if $\inf \left\{\delta: \#\left\{(p, q) \in \mathbb{N} \times \mathbb{N}^{*}:|\alpha-p / q| \leq q^{-\delta}\right\}=\infty\right\}=2$.

- Poisson point processes.

Let $S$ be a Poisson point process [25] with intensity $\lambda \otimes \nu$ in the square $[0,1] \times(0,1]$, where $\lambda$ denotes the Lebesgue measure on $\mathbb{R}$ and $\nu$ is a positive locally finite Borel measure on $(0,1]$. Let us take the family $\left\{\left(x_{n}, l_{n}\right)\right\}_{n \geq 1}$ equal to the set $S$. Let

$$
\beta_{j}=j^{-1} \log _{c} \nu\left(\left(2^{-(j-1)}, 2^{-(j-2)}\right]\right) \text { and } \beta=\limsup _{j \rightarrow \infty} \beta_{j} .
$$

Proposition 6.2.

(i) Suppose $\int_{[0,1]} \exp \left(2 \int_{[t, 1]} \nu((2 y, 1)) d y\right) d t=+\infty$. This implies in particular $\beta \geq 1$. With probability 1, (1.8) holds.

(ii) $\left\{\left(x_{n}, \lambda_{n}\right)\right\}_{n \geq 1}$ is WRA family almost surely if and only if $\beta \leq 1$.

The first part of this proposition follows from Shepp's theorem $([\mathbf{2 8}])$. The second part follows from the proofs of Lemma 5 and 8 of $[\mathbf{2 2}]$, and some arguments provided in $[\mathbf{3}]$.

\section{Proof of Proposition $2 \cdot 2$}

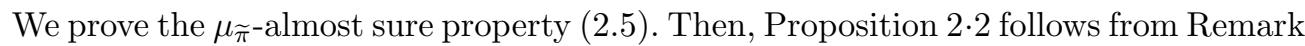
$2 \cdot 3$. Let us first introduce some notations and properties.

For $n \geq 0$ let $\Sigma_{n}=\{0, \ldots, b-1\}^{n}$, where $\Sigma_{0}$ contains only the empty word denoted by $\emptyset$. Also let $\Sigma^{*}=\bigcup_{n \geq 0} \Sigma_{n}$ and $\Sigma=\{0, \ldots, b-1\}^{\mathbb{N}^{*}}$. The concatenation operation from $\Sigma^{*} \times\left(\Sigma^{*} \bigcup \Sigma\right)$ to $\left(\Sigma^{*} \bigcup \Sigma\right)$ is denoted . 
For $x \in \Sigma$ and $n \geq 1$, let $(x \mid n)$ be the projection of $x$ on $\Sigma_{n}$. Then for $n \geq 1$ and $w \in \Sigma_{n}$, we set $[w]=\{x \in \Sigma:(x \mid n)=w\}$. Given two words of infinite length $x, y \in \Sigma$, we define $x \wedge y$ as $\left(x \mid n_{0}\right)$, where $n_{0}=\sup \{n \geq 1:(x \mid n)=(y \mid n)\}$. We adopt the convention that $\inf \emptyset=0$ and $(x \mid 0)$ is the empty word $\emptyset$.

The length of any element $w$ of $\Sigma_{n}$ is equal to $n$ and is denoted by $|w|$.

For $n \geq 1$, we denote by $r^{\cdot n}$ (resp. $\ell^{\cdot n}$ ) the element of $\Sigma_{n}$ consisting of $n$ times the digit 0 (resp. $b-1$ ). Finally, let $f_{b}$ be the mapping $f_{b}: x \in \Sigma \mapsto \sum_{n \geq 1} x_{n} b^{-n} \in[0,1]$.

The measure $\mu_{\widetilde{\pi}}$ is the image by $f_{b}$ on $[0,1]$ of the measure $\nu_{\widetilde{\pi}}=\rho^{\otimes \mathbb{N}^{*}}$ on $\Sigma$, where $\rho=\sum_{i=0}^{b-1} \pi_{i} \delta_{i}$ is an atomic measure $\left(\delta_{i}\right.$ is a Dirac mass at $\left.i\right)$.

For every $i \in\{0, \ldots, b-1\}$, the application of the law of iterated logarithm to the sequence of i.i.d random variables $\left(X_{k}(x)=\mathbf{1}_{i}\left(x_{k}\right)\right)_{k \geq 1}$ relatively to $\nu_{\tilde{\pi}}$ shows that for $\nu \tilde{\pi}$-almost every $x$, we have

$$
\limsup _{n \rightarrow \infty} \frac{\left|\widetilde{\phi}_{i, n}(x)-\pi_{i}\right|}{\sqrt{n^{-1} \log \log (n)}}=\sqrt{\pi_{i}\left(1-\pi_{i}\right)} \leq 1 / 2 \text {, where } \widetilde{\phi}_{i, n}(x)=\frac{\sum_{1 \leq k \leq n} X_{n}(x)}{n} .
$$

For every $n \geq 1$ and $y \in(0,1)$ not a $b$-adic number, there exists a unique element $x \in \Sigma$ such that $y=f_{b}(x)$. Moreover, with any triplet of intervals $\left(I_{n, k}\right)_{k:\left|k-k_{n, y}^{b}\right| \leq 1}$ is naturally associated a unique triplet of words $\left(w_{-1}, w_{0}, w_{1}\right) \in \Sigma_{n}^{3}$ such that $f\left(\left[w_{\epsilon}\right]\right)=I_{n, k_{n, y}^{b}+\epsilon}$, with $w_{0}=(x \mid n)$.

For $\epsilon \in\{-1,0,1\}$, we denote $(x \mid n)^{\epsilon}=w_{\epsilon}\left(\right.$ thus $\left.(x \mid n)^{0}=(x \mid n)\right)$. It is easily seen that by construction, for $\epsilon \in\{-1,0,1\}$,

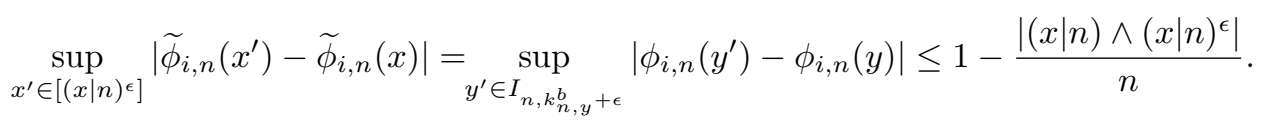

Lemma 7·1. There exists $K>0$ such that for each $\epsilon \in\{-1,1\}$, for $\nu_{\widetilde{\pi}}$-almost every $x$, for $n$ large enough, $\left|(x \mid n) \wedge(x \mid n)^{\epsilon}\right| \geq n-K \log (n)$.

Proof. Let us deal with the case $\epsilon=-1$, the case $\epsilon=1$ is identical. The mapping $x \in[0,1] \mapsto\left((x \mid n)^{-1},(x \mid n)\right)$ takes its values in the set $\left(u=(x \mid n)^{-1} \wedge(x \mid n)\right)$

$$
\bigcup_{k=0}^{n-1}\left\{\left(u \cdot i \cdot \ell^{\cdot(n-k+1)}, u \cdot(i+1) \cdot r^{\cdot(n-k+1)}\right): u \in \Sigma_{k}, i \in\{0, \ldots, b-2\}\right\},
$$

Moreover, the mapping $x^{\prime} \mapsto\left(\left(x^{\prime} \mid n\right)^{-1},\left(x^{\prime} \mid n\right)\right)$ is constant over $[x \mid n]$. Hence, given any integer $g(n) \in[0, n-1]$, we have (recall that $\pi_{0}$ is the first coordinate of $\tilde{\pi} \in[0,1]^{b}$ )

$$
\begin{aligned}
\nu_{\tilde{\pi}}\left(\left\{x:\left|(x \mid n)^{-1} \wedge(x \mid n)\right| \leq g(n)\right\}\right) & \leq \sum_{k=0}^{g(n)} \sum_{u \in \Sigma_{k}} \sum_{i=0}^{b-1} \mu_{\widetilde{\pi}}\left(\left[u \cdot(i+1) \cdot r^{\cdot(n-k+1)}\right]\right) \\
& \leq b \sum_{k=0}^{g(n)} \pi_{0}^{n-k+1}=O\left(\pi_{0}^{n-g(n)}\right) .
\end{aligned}
$$

If we take $g(n)=\left[n+\frac{2}{\log \left(\pi_{0}\right)} \log (n)\right]$, then the last inequality yields that the sum $\sum_{n \geq 1} \nu_{\widetilde{\pi}}\left(\left\{x:\left|(x \mid n)^{-1} \wedge(x \mid n)\right| \leq g(n)\right\}\right)$ is finite.

The Borel-Cantelli lemma ends the proof.

Remark that $\nu_{\widetilde{\pi}}\left(\left[x^{\prime} \mid n\right]\right)=\prod_{i=0}^{b-1} \pi_{i}^{n \widetilde{\phi}_{i, n}\left(x^{\prime}\right)}$, for any $x^{\prime} \in \Sigma$. Combining this remark with (7.1), (7.2) and Lemma 7.1, (2.5) is proved, as well as Proposition 2.2. 


\section{REFERENCES}

[1] A.Baker, W.M. Schmidt, Diophantine approximation and Hausdorff dimension. Proc. London Math. Soc. 21 (1970), 1-11.

[2] J. Barral, S. Seuret, Sums of Dirac masses and conditioned ubiquity. C. R. Acad. Sci. Paris, Ser. I 339 (2004), 787-792.

[3] J. BARral, S. SEURet, Heterogeneous ubiquitous systems and Hausdorff dimension in $\mathbb{R}^{d}$. Arxiv math.GM/0503419.

[4] V. Beresnevich, H. Dickinson, S. Velani, Measure theoretic laws for limsup sets, Memoirs of the AMS., vol. 179, number 840 (2006) 1-91.

[5] V.I. BERNIK, K.I. TISHChENKO, Integral polynomials with an overfall of the coefficient values and Wirsing's theorem. Dokl. Akad. Nauk Belarusi 37 (1993), 9-11.

6] A.S. Besicovitch, On the sum of digits of real numbers represented in the dyadic system, Math. Ann 110 (1934), 321-330.

[7] G. Brown, G. Michon, J. Peyrière, On the multifractal analysis of measures. J. Stat. Phys. 66 (1992), 775-790.

[8] Y. Bugeaud, An inhomogeneous Jarnik theorem. J. Anal. Math. 92 (2004), 327-349 .

[9] Y. Bugeaud, Intersective sets and Diophantine approximation, Mich. Math. J. 52 (2004), 667-682.

[10] Y. Bugeaud, O. TeuliÉ, Approximation d'un nombre réel par des nombres algébriques de degré donné. Acta Arith. 93 (2000), 77-86.

[11] J.W.S. Cassels, An Introduction to Diophantine Approximation. Cambridge University Press (1957)

[12] M.M. Dodson, Exceptional sets in dynamical systems and Diophantine approximation. Rigidity in Dynamics and Geometry (Cambridge, 2000), 77-98, Springer, Berlin (2002).

[13] M.M. Dodson, B.P. Rynne, J.A.G.Vickers, Diophantine approximation and a lower bound for Hausdorff dimension. Mathematika 37 (1990), 59-73.

[14] M.M. Dodson, M.V. Melián, D. Pestana, S.L. VÉlani, Patterson measure and Ubiquity. Ann. Acad. Sci. Fenn. Ser. A I Math. 20 (1995), 37-60.

[15] H. Eggleston, The fractonial dimension of a set defined by decimal properties. Quart. J. Math. Oxford Ser. 20 (1949), 31-36.

[16] K.J. FALCONER, Classes of sets with large intersection, Mathematika 32 (1985), 191-205.

[17] K.J. FAlCONER, Sets with large intersection properties. J. London Math. Soc. (2)(49) (1994), 267-280.

[18] K.J. Falconer, Techniques in Fractal Geometry. Wiley, New York (1997).

19] K.J. FAlCONER, Representation of families of sets by measures, dimension spectra and Diophantine approximation, Math. Proc. Camb. Phil. Soc. 128 (2000), 111-121.

[20] G.H. Hardy, E.M. Wright, An introduction to the theory of numbers. Oxford Univ. Press, NY (1978).

[21] J.E. Hutchinson, Fractals and self-similarity, Indiana Univ. Math. J. 30 (1981), 713-747.

[22] S. Jaffard, The multifractal nature of Lévy processes. Probab. Theory Relat. Fields, 114 (1999), 207-227.

[23] S. Jaffard, On lacunary wavelet series. Ann. Appl. Prob. 10 (2000), 313-329.

[24] V. JARNIK, Diophantischen Approximationen und Hausdorffsches Mass. Mat. Sbornik 36 (1929), 371-381.

[25] J.F.C Kingman, Completely random measures. Pacific J. Math., 21 (1967), 59-78.

[26] J. Levesley, C. Salp, S. Velani, On a problem of K. Mahler: Diophantine approximation and Cantor sets, preprint.

[27] P.Mattila, Geometry of Sets and Measures in Euclidian Spaces. Cambridge Studies in Advanced Mathematics, Cambridge University Press (1995).

[28] L.A. Shepp, Covering the line with random intervals. Z. Wahrscheinlichkeitstheorie und Verw. Gebiete 23 (1972), 163-170. 